\title{
VIRTUDES EPISTÉMICAS REFLEXIVAS. EL CONOCIMIENTO COMO UNA CUESTIÓN DE CONFIANZA ${ }^{1}$
}

\author{
REFLECTIVE EPISTEMIC VIRTUES. \\ KNOWLEDGE AS A MATTER OF TRUST
}

\author{
Manuel Liz
}

\begin{abstract}
RESUMEN
De acuerdo a Ernest Sosa, el conocimiento reflexivo debería ser capaz de integrar algunas circularidades epistémicas como fuentes virtuosas de conocimiento. Argumentaremos que tal conocimiento reflexivo tiene que estar basado en ciertas capacidades para delegar y aplazar de manera adecuada la justificación de nuestras creencias de primer orden. También argumentaremos que entender esas capacidades comunitarias y temporales como constituyendo virtudes epistémicas reflexivas nos conduce fuera de cualquier concepción criterial del conocimiento. Para estas concepciones, conocer siempre requiere saber que se han satisfecho determinados criterios. Si el conocimiento propiamente humano inevitablemente necesita alguna dosis de reflexión, y si nuestro conocimiento reflexivo necesariamente depende del ejercicio virtuoso de ciertas capacidades para delegar y aplazar la justificación, entonces en último término el conocimiento no puede ser criterial. El conocimiento humano es más bien una cuestión de confianza
\end{abstract}

PALABRAS CLAVE: Conocimiento; criterios; perspectiva epistémica; circularidad epistémica; conocimiento animal; conocimiento reflexivo; ascenso epistémico; virtudes epistémicas reflexivas; delegación de la justificación; aplazamiento de la justificación.

\begin{abstract}
According to Ernest Sosa, reflective knowledge would have to be able to integrate some epistemic circularities as virtuous sources of knowledge. We will argue that such reflective knowledge has to be based on some capacities for delegating and relegating in adequate ways the justification of our first-order beliefs. Also, we will argue that
\end{abstract}

\footnotetext{
${ }^{1}$ Este trabajo se han llevado a cabo en el contexto del Proyecto de Investigación, FFI201457409, financiado por el Ministerio de Economía y Competitividad del Gobierno de España.
} 
to understand those communitarian and temporal capacities as constituting reflective epistemic virtues leads us outside any criterial conception of knowledge. For these conceptions, knowing always requires to know that certain criteria are fulfilled. If human knowledge worth of the name unavoidably needs some amount of reflection, and if our reflective knowledge necessarily depends on virtuous delegation and deferring, then at the end of the day knowledge cannot be criterial. Human knowledge is rather a matter of trust.

KEYWORDS: Knowledge; criteria; epistemic perspective; epistemic circularity; animal knowledge; reflective knowledge; epistemic ascent; reflective epistemic virtues; delegation of justification; deferring of justification.

En este trabajo quiero explorar algunos senderos que parten del camino conceptual emprendido por la epistemología de Ernest Sosa. Más concretamente, quiero explorar el abandono general de una concepción criterial del conocimiento según la cual conocer siempre requiere saber que se han satisfecho determinados criterios. Más allá del simple conocimiento animal, que suponemos puede desarrollarse al margen de la anterior exigencia criterial, tal abandono general sería consecuencia de la necesidad de tener en cuenta ciertas virtudes epistémicas reflexivas muy especiales, estrechamente vinculadas a la necesidad de asumir perspectivas globales sobre nuestra posición epistémica en el mundo integrando amplias circularidades virtuosas. Esas virtudes epistémicas reflexivas estarían basadas en nuestra capacidad para conocer mediante la delegación en otros sujetos de nuestra comunidad epistémica y mediante el aplazamiento temporal.

Los primeros apartados ( 1 y 2 ) presentan esta problemática de un modo muy general. A continuación (apartados 3 y 4), me adentraré en la obra de Sosa discutiendo algunos detalles. Propondré después (apartado 5) una concepción no-criterial del conocimiento basada en el ejercicio virtuoso de nuestras capacidades para delegar y aplazar reflexivamente la justificación. El trabajo concluye (apartado 6) con unas breves conclusiones.

\section{CONOCIMIENTO ANIMAL, CONOCIMIENTO REFLEXIVO Y CRITERIALIDAD}

La distinción que Sosa hace en múltiples lugares de su obra entre "conocimiento animal" y "conocimiento reflexivo" es sumamente importante. Es fácil asumir que el ámbito del conocimiento animal no sea criterial. La base de nuestro conocimiento consistiría en un conjunto de capacidades cognitivas que se ma- 
nifiestan de manera natural, sin requerir ascensos epistémicos por parte de los sujetos que poseen tal conocimiento. Se puede conocer en este sentido animal sin saber que se han satisfecho criterios, lo cual para una concepción criterial del conocimiento implicaría que se puede conocer en este sentido sin saber que se conoce. Esto parece una trivialidad. Pero no lo es en absoluto. Y ofrece un fundamento bastante firme para nuestro conocimiento. Un fundamento que se hace más sólido al recibir la iluminación de un conocimiento reflexivo que evalúa e incorpora ese conocimiento animal en una perspectiva global sobre nuestra posición epistémica en la realidad. Tal perspectiva inevitablemente involucrará grandes dosis de conocimiento animal. Y con ello, surgirán numerosas circularidades epistémicas. Sin embargo, no todas ellas son viciosas. Las promesas de amplitud y de riqueza estructural en el contenido de muchas de estas circularidades hacen que deban ser consideradas fuentes virtuosas de conocimiento.

El conocimiento reflexivo lleva a cabo ascensos epistémicos. Busca conocer que conocemos. Y promete conseguirlo a través de amplias circularidades y de perspectivas globales. Con ello, el conocimiento reflexivo lleva a otro nivel nuestro trasfondo de conocimiento animal. Empleando de nuevo una metáfora clásica, podemos decir que lo ilumina reflexivamente. El problema que quiero abordar es el de si hacer todo esto implica a su vez saber que se han satisfecho ciertos criterios. En el conocimiento reflexivo hacemos uso de nuestro conocimiento animal. Y como hemos dicho, el conocimiento animal no es criterial. Pero, zacaba cubriéndolo todo el manto de una criterialidad reflexiva que impone tentativamente criterios, evalúa su grado de satisfacción, vuelve a ensayar nuevos criterios, vuelve a evaluar su satisfacción, etc., en un largo e indefinido "etc." que nos constituye?

Cabe introducir nuestra inquietud desde el viejo problema del criterio. ${ }^{2}$ Este problema nos enfrenta a tres opciones: el escepticismo, el fundamentalismo y el coherentismo. Y podemos preguntar, ¿acaba este problema recibiendo en Sosa una compleja solución coherentista? La circularidad y la perspectiva, una perspectiva amplia capaz de integrar como virtuosas algunas circularidades, ¿constituye algo más que una propuesta coherentista muy elaborada?

¿No hay más factores relevantes? Creo que sí los hay. Las circularidades virtuosas remiten a una justificación que implica delegaciones en una comunidad epistémica y desarrollos futuros de las actividades epistémicas que caen bajo nuestra perspectiva. Estos factores comunitarios y temporales son decisivos en el conocimiento reflexivo. Y si los tenemos en cuenta seriamente, podremos apreciar que

\footnotetext{
${ }^{2}$ Véase Chisholm (1982) y Chisholm (1989).
} 
nuestro conocimiento reflexivo acaba inclinándose hacia la no-criterialidad. Así pues, la propuesta de Sosa no sería coherentista porque no se ve afectada por el problema del criterio. Su juego es otro muy distinto. En último término, nuestro conocimiento no consiste en saber que se han satisfecho criterios. Consiste más bien en algo como "mantener una confianza". O al menos, esta es la idea que quiero explorar.

Habría una diferencia crucial entre la no-criterialidad del conocimiento animal y la no-criterialidad última del conocimiento reflexivo. Mientras que la primera puede interpretarse reflexivamente como un tipo de fundamentalismo que ofrece un tipo de solución interesante al problema del criterio, un tipo de solución que en la epistemología de Sosa estaría en plena sintonía con el reciente confiabilismo, un tipo de solución que además resultaría compatible con las exigencias del conocimiento reflexivo, la no-criterialidad a la que nos conduce el conocimiento reflexivo no puede ser interpretada de este modo. ¿Por qué no? Hay una razón muy clara. La no-criterialidad del conocimiento reflexivo sólo podría ser interpretada en los mismos términos en los que es interpretada la no-criterialidad del conocimiento animal desde un nuevo conocimiento reflexivo que volvería una y otra vez a apartarnos de la criterialidad.

$\mathrm{El}$ anterior argumento tiene un gran peso. No podemos hacer de los factores comunitarios y temporales del conocimiento reflexivo un nuevo fundamento. Las capacidades para delegar y aplazar la justificación no pueden ser reflexivamente consideradas un fundamento de nuestro conocimiento. No pueden ser consideradas de ese modo porque considerarlas reflexivamente así implicaría ya delegar y aplazar la justificación en un sentido que se situaría muy al margen de la constatación de que se han satisfecho ciertos criterios.

Queremos que nuestras vidas sean "vidas examinadas". Y queremos que nuestro conocimiento también lo sea. Sólo así merece la pena. Y sólo así merecerá nuestra confianza. Pero al buscar la iluminación reflexiva, nuestro conocimiento acaba siendo algo profundamente no-criterial.

La estructura de nuestro conocimiento es curiosa. Comienza siendo no-criterial en forma de un conocimiento animal. A partir de esos inicios, se desarrolla hasta cierto punto en un ambiente reflexivo de criterialidad. Pero al final, acaba volviendo a ser no-criterial. Aunque en niveles intermedios conocer implique saber que se han satisfecho determinados criterios, en último término nuestro conocimiento, un conocimiento humano que aspira a saber que se conoce, no consiste en saber que se han satisfecho ciertos criterios supuestamente pertinentes. Ni siquiera consiste en saber que se han satisfecho criterios tentativos que se van proponiendo y re-inventando según se desarrolla el propio conocimiento. 
Nuestras capacidades cognitivas animales son iluminadas por la reflexión. Y el marco final de nuestro conocimiento reflexivo es una justificación que se apoya en capacidades comunitarias y temporales. Como hemos dicho, esto último no ofrece ningún fundamento. No puede hacerlo. Lo que más bien ocurre es que acabamos saliéndonos del juego del conocimiento criterial.

Sin embargo, también hay vida epistémica fuera del juego criterial. Si la suerte no nos es del todo adversa, esa justificación basada en capacidades comunitarias y temporales tal vez pueda darnos toda la confianza epistémica que necesitamos. ${ }^{3}$

\section{EPISTEMOLOGÍA, CONFIANZA Y CRITERIOS}

La epistemología es la expresión conceptual de una obsesión. Nos obsesiona la confianza que merecen nuestras creencias. En general, se busca reafirmar esa confianza. Pero a veces, también se busca socavarla. El escepticismo nos enseña a no ser confiados en exceso. Mientras que otras perspectivas epistemológicas conceden créditos con facilidad, el escepticismo sólo concede crédito epistémico con avales completamente seguros. Y esto, rara vez sucede. En cualquier caso, nos preocupa la seguridad que debemos sentir ante nuestras creencias sobre el mundo que nos rodea. Y la seguridad que debemos sentir ante nuestras creencias sobre nosotros mismos. $\mathrm{Al}$ menos, queremos que sea alta la probabilidad de que el mundo sea como nos lo pintan nuestras mejores creencias. Y que también sea alta la probabilidad de que seamos como nuestras mejores creencias sobre nosotros mismos nos dicen que somos. ${ }^{4}$

\footnotetext{
${ }^{3}$ Pueden encontrarse muy buenos análisis de la suerte, o azar, en nuestras atribuciones morales y epistémicas, y de su papel tremendamente contextual y perspectival, en Hales (2015a), Hales (2015b) y Hales (2016). Es imposible descartar la intervención de la suerte, o del azar, tanto en nuestra contra como a favor nuestro. Sólo un conocimiento completo podría hacerlo. Pero, justamente, la posibilidad de conocimiento implica que la suerte o el azar no intervienen de manera relevante. La posibilidad de ofrecer una solución satisfactoria a los casos tipo Gettier también depende de asumir que podemos descartar la intervención de la suerte o del azar. Hay aquí otra compleja familia de circularidades interesantes. Toda atribución de confianza epistémica implica tener una mínima confianza en que el azar, o la suerte, no son componentes determinantes. Sin embargo, muchas veces tenemos esa confianza. Y entonces podemos decir que estamos muy cerca del conocimiento. Algo muy parecido ocurre en otros campos. Para bien o para mal, las presunciones de suerte, o de azar, pueden interferir en nuestras atribuciones de estados y contenidos mentales, en nuestras atribuciones de acciones intencionales, en nuestras atribuciones de valor moral y estético, etc.

${ }^{4}$ Esta noción de seguridad estaría muy cerca de lo que Sosa ha llamado "safety". Véase Sosa (2008) y DeRose (2008). Véase también la nota 12. Sosa insiste en que la seguridad debe dis-
} 
Los problemas epistemológicos siempre son una cuestión de confianza. Y muchas epistemologías han visto frustrados sus esfuerzos por llegar a evaluar convincentemente la confianza que merecen nuestras creencias. La epistemología de Sosa ofrece algo nuevo. Aunque nos sean familiares los ingredientes empleados, la combinación propuesta es tremendamente nueva y sugerente. Y merece la pena probarla. Pero al final, el resultado de tal degustación puede ser tan inesperado como fascinante. En este trabajo intentaré mostrar que es así respecto de algunos rasgos esenciales de nuestro conocimiento.

La epistemología de Sosa nos habla de una distinción fundamental entre conocimiento animal y conocimiento reflexivo, del virtudes epistémicas que se activan y mejoran en el desarrollo del conocimiento, de aptitudes que conectan el hecho de tener ciertas creencias con la verdad de su contenido, de algunas circularidades epistémicas que pueden dejar de ser viciosas para convertirse en fuentes inevitables de conocimiento reflexivo, de la necesidad de una perspectiva coherente y equilibrada sobre nuestra posición epistémica en la realidad, etc. Creo que cuando se consideran seriamente todas estas cosas, nos vemos finalmente llevados fuera de los límites de una concepción simplemente criterial del conocimiento.

Según Sosa, el conocimiento animal no necesita saber que se han satisfecho criterios. El conocimiento animal simplemente se tiene. No debe ser evaluado como tal conocimiento por los sujetos que lo poseen. Ni, tampoco, debe ser evaluado así antes de poseerlo. Por supuesto, podemos evaluarlo reflexivamente. De hecho, lo hacemos muchas veces. Y obviamente, lo hace la epistemología. Pero si tenemos conocimiento animal, lo tenemos con independencia de toda reflexión. El conocimiento animal no necesita ningún ascenso epistémico. Y como consecuencia de ello, no es un conocimiento criterial.

tinguirse de la sensibilidad (sensitivity). En términos de probabilidad, ambas nociones podrían definirse así:

Seguridad: Si se creyera que $p$, entonces sería muy probable que fuera verdad que $p$.

Sensibilidad: Si no fuera verdad que $p$, entonces sería muy probable que no se creyera que $p$.

La distinción entre estas dos nociones se basa en la no contraposición de los condicionales subjuntivos (probabilísticos, en nuestro caso). Se pueden tener creencias muy seguras en el anterior sentido, por ejemplo creencias "mooreanas", aunque no puedan descartarse las conocidas posibilidades escépticas sobre el mundo externo. Acerca de este punto, véase también Zalabardo (2014). La única diferencia entre la noción de seguridad empleada por Sosa y el uso que estoy sugiriendo de esta noción consistiría en que, de acuerdo a mi uso, la seguridad de nuestras creencias no sería tanto algo que las creencias deban tener, como sobre todo algo que nosotros debemos sentir que las creencias tienen. Por mucho que una creencia sea segura, esto no tiene ninguna eficacia epistémica reconocible como conocimiento humano si no se siente esa seguridad. 
La criterialidad es una forma de llevar a cabo ascensos epistémicos. De manera más precisa, podemos decir que un conocimiento es criterial si se cumplen las siguientes dos condiciones:

1) La existencia de tal conocimiento implica que los propios sujetos que lo poseen lo han cualificado como tal conocimiento en virtud de la satisfacción de ciertos criterios.

2) La existencia de tal conocimiento implica que se ha cualificado como tal conocimiento en virtud de la satisfacción de ciertos criterios antes de dicha posesión.

Resulta claro que por sí mismo el conocimiento animal no es un conocimiento criterial. Podemos llamarlo conocimiento no-criterial. Tanto una reflexión de tipo fundamentalista como incluso una reflexión de tipo coherentista parecen poder convertirlo en conocimiento criterial. Ahora bien, podemos preguntar, ¿ ¿erá criterial el propio conocimiento reflexivo? Lo peculiar del conocimiento reflexivo es el ascenso epistémico. El conocimiento reflexivo es un conocimiento que quiere conocer que conoce. Aparentemente, esto nos introduce de lleno en el problema del criterio. Y consecuentemente, si rechazamos el escepticismo, esto haría del propio conocimiento reflexivo un conocimiento también criterial. Sin embargo, voy a argumentar que, en última instancia, tal vez también el conocimiento reflexivo sea un conocimiento no-criterial. Y ésta vez, en un sentido mucho más radical.

El punto crucial es el siguiente. Al considerar seriamente la combinación ofrecida por Sosa, el conjunto de lo que debemos aceptar como virtudes epistémicas se agranda sorprendentemente. El conocimiento reflexivo acaba sustentándose (autosustentándose) en amplias circularidades virtuosas capaces de añadir justificación a nuestras creencias y perspectivas globales sobre nuestra posición epistémica en la realidad. Y en estos terrenos, no basta ya con un buen ejercicio de nuestras capacidades de percepción, memoria, razonamiento, etc. Ni siquiera basta con un buen ejercicio de nuestra capacidad para obtener conocimiento por testimonio. Cuando se considera la necesidad de que ciertas circularidades epistémicas amplias puedan llegar a ser virtuosas y cuando se considera la necesidad de obtener perspectivas globales sobre nuestra posición epistémica en la realidad, resulta inevitable adoptar en nuestro conocimiento reflexivo puntos de vista que sean comunitarios y también puntos de vista que sean temporales.

Más concretamente, resulta inevitable apelar a la existencia de justificaciones epistémicas que podemos llamar "delegadas" y "aplazadas". Se trata de justificaciones que deben dejarse pendientes, en un sentido comunitario o temporal. Son justificacio- 
nes epistémicas que deberían ser llevadas a cabo o bien por otros sujetos distintos de nosotros, o bien por alguien (acaso nosotros mismos) en un futuro más o menos lejano. En estas variedades de justificación epistémica se podría obtener justificación a través de un ejercicio virtuoso de nuestra capacidad para delegar y de nuestra capacidad para aplazar. Y esas justificaciones nos permitirían saber que conocemos. Harían plausibles ciertas atribuciones de conocimiento reflexivo "aquí" y "ahora". Conoceríamos reflexivamente "aquî" y "ahora" que conocemos porque somos capaces de "delegar y aplazar con confianza".

Estamos ante un ascenso epistémico al margen de la criterialidad. Y no se trata únicamente de que nuestro conocimiento funcione de hecho así. No puede funcionar de otra forma. Si es posible para nosotros el conocimiento, ha de ser un conocimiento cuya justificación reflexiva se apoye en nuestras capacidades para delegar y para aplazar. Esto es lo que nos sitúa fuera de una concepción criterial del conocimiento. No sólo nuestro conocimiento animal se desarrolla al margen de la necesidad de saber que se han satisfecho criterios. En último término, también debe hacerlo nuestro conocimiento más reflexivo.

¿Qué significa abandonar una concepción criterial del conocimiento? Significa que en último término, allí donde hemos emprendido arduos procesos de ascenso epistémico, conocer no consiste en saber que se han satisfecho criterios. No consiste en disponer de reglas, procedimientos, métodos, etc., para eliminar "todas las manzanas malas" del cesto de nuestras creencias antes de poder pronunciarnos sobre cuáles de ellas constituyen conocimientos. Ni siquiera consiste en disponer de reglas, procedimientos, métodos, etc., provisionales y tentativos, que vayamos mejorando sobre la marcha. Es importante apreciar que también queda descartada esta variedad de "criterialidad provisional y tentativa". Conocer acaba consistiendo en disponer de una base adecuada para mantener un grado alto de confianza en nuestras creencias.

Esto tiene ventajas. Si abandonamos la concepción criterial del conocimiento, entonces dejamos atrás el llamado "problema del criterio" y el trilema al que nos aboca (o escepticismo, o fundamentalismo, o coherentismo). Si en última instancia conocer no consiste en saber que se han satisfecho criterios, entonces no habrá ningún conjunto de criterios últimos que debamos justificar. ¡Ni siquiera algo tan vago y general como la racionalidad. Puede haber criterios (reglas, procedimientos, métodos, etc.) en múltiples fases de nuestro conocimiento, y un gran número y variedad de ellos, pero no criterios de última instancia.

Dejar atrás el problema del criterio también implica evitar otro problema importante que ha sido recientemente denominado "problema del conocimiento 
fácil". Si respondemos al problema del criterio asumiendo ciertos tipos de conocimientos fundamentalistas, entonces a partir de ellos y mediante inferencias sencillas parece que podríamos llegar muy fácilmente a conclusiones sobre el carácter verídico de nuestras facultades, en general y en casos particulares, y con ello también a conclusiones fáciles acerca de que no se dan determinados escenarios escépticos, nuevamente en general y en casos particulares. Y sin embargo, intuitivamente, esos conocimientos parecen demasiado sustantivos como para ser obtenidos de esa manera tan fácil. ${ }^{5}$ No me extenderé en este punto. Es obvio que sin criterialidad los enfoques que podemos dar a este problema cambian por completo.

Pero, más concretamente, ¿en qué puede consistir el conocimiento al margen de la criterialidad? Hay muchas formas de apartarse de una concepción criterial del conocimiento. El pragmatismo y el utilitarismo, por ejemplo, ofrecen concepciones nocriteriales del conocimiento. También, la concepción del conocimiento como algo últimamente indefinible. ${ }^{6} \mathrm{Y}$ no son las únicas posiciones filosóficas que lo hacen.

Las concepciones no-criteriales del pragmatismo y del utilitarismo no tienen ninguno de los problemas anteriores. $\mathrm{Ni}$ se ven afectadas por el problema de criterio ni por el problema del conocimiento fácil. Y tampoco tiene estos problemas una concepción que haga del conocimiento algo indefinible. Sin embargo, en todas ellas surge otro problema no menos grave. En ninguna de estas concepciones nocriteriales conseguimos entender el conocimiento justamente como conocimiento. Este problema puede plantearse de un modo muy directo. ¿Por qué ese estado indefinible, un estado que incluso implica creencia, verdad y justificación, ha de ser propiamente un estado de conocimiento? ¿Y por qué es conocimiento lo que entienden el pragmatismo y el utilitarismo por conocimiento? Realmente, no está nada claro.

Se impone una pregunta. ¿Es posible una concepción no-criterial del conocimiento que, esquivando el problema del criterio y el problema del conocimiento fácil, permita también entender el conocimiento justamente como conocimiento, y como un conocimiento que en el caso humano aspira a saber que conoce? ¿Es posible una concepción no-criterial que permita entenderlo en un sentido parecido a como nos prometen entenderlo las concepciones criteriales? Creo que sí. Y que podemos llegar a verla desde lejos al final del camino epistemológico de Ernest Sosa. Intentaré mostrar que podemos llegar a ver esa concepción al final de las circularidades virtuosas y de las perspectivas reflexivas.

\footnotetext{
${ }^{5}$ Sobre el problema del conocimiento fácil (The problem of easy knowledge), véase Sosa (2009, cap. 10) y Zalabardo (2005). Cohen (2002) inició esta problemática.

${ }^{6}$ Williamson (2000) constituye la principal exposición y defensa de esta posición.
} 
Yo diría que nuestras creencias se acercan al conocimiento cuando muy difícilmente podríamos llegar a perder la confianza que depositamos en ellas. Podríamos caracterizar la aproximación al conocimiento como una fuerte confianz̧a epistémica que se mantiene.

El que el conocimiento sea una cuestión de confianza explica porqué es tan difícil definirlo. ¿Qué es la confianza epistémica? ¿Qué es, en general la confianza? Sí, en cierto sentido es un estado intencional. Es más, tiene asociados conjuntos característicos de causas y efectos. Pero la confianza también es un estado cualitativo. Sin esto último, lo más que tenemos son simulacros de confianza. Y el problema ahora nos es ya conocido: ¿cómo definir un estado cualitativo?

El que el conocimiento sea una cuestión de confianza, también explica que pueda llegar a intervenir de manera tan desconcertante el azar, o la suerte, haciendo que perdamos la confianza. Siempre pueden estar organizadas las cosas de manera que la justificación de nuestras creencias se conecte con su verdad únicamente por az̧ar (llamémoslo también suerte). Y sospechar esto, claro que hace que perdamos la confianza. Sin embargo, sospechar también requiere confiar en ciertas cosas. Y en ese juego de confianzas, la sospecha no siempre gana. ¿Cómo podría, por ejemplo, sospechar de algo si al mismo tiempo sospecho también de mi capacidad para conocer el contenido de mi sospecha? La sospecha, la pérdida de confianza, tiene límites. Y estos límites configuran los límites del escepticismo.

Yo diría que el conocimiento se parece a un sistema de crédito. Y que el crédito último que concedemos a nuestras creencias se basa en la delegación y en el aplazamiento. El conocer reflexivamente que conocemos, respetando cualquier necesidad de ascenso epistémico, se basa en la delegación y en el aplazamiento. Las justificaciones por delegación y por aplazamiento consiguen el crédito epistémico con el que recuperamos la confianza en nuestras creencias. Sin embargo, lo hacen de manera comisionada y a largo plazo. La justificación escrupulosamente criterial de nuestras creencias siempre queda pendiente.

¿Se podrá acabar saldando ese crédito epistémico? Tal vez no se pueda. Es muy posible que no pueda llegar a saldarse nunca el crédito que sustenta la confianza en el conjunto de nuestra actividad epistémica. Y no obstante, disponemos de ese crédito. Pues confiamos en nuestras creencias obtenidas con esfuerzo. 


\section{ERNEST SOSA: UNA EPISTEMOLOGÍA BASADA EN LA VIRTUD}

Ernest Sosa es actualmente el representante más destacado de la corriente epistemológica conocida como "virtue epistemology", que en castellano podemos denominar "epistemología de la virtud", o mejor aún "epistemología basada en la virtud". La idea fundamental de este enfoque es que la fuente última de los valores y normas epistémicamente relevantes, es decir, de cosas como la justificación, la racionalidad, el estatus epistémico, o la propia definición de lo que ha de contar como conocimiento, está en ciertas disposiciones poseídas por los sujetos y las comunidades epistémicas en sus respectivos entornos materiales y contextos intencionales. Esas disposiciones son determinadas capacidades psicológicas, competencias cognitivas, aptitudes, habilidades, rasgos de carácter, etc. En general, pueden llamarse "virtudes epistémicas".

Cabe hacer un rápido bosquejo de este enfoque epistemológico destacando su capacidad para marcar distancias frente a otros enfoques. Una epistemología basada en la virtud tiene perfiles sustantivos muy característicos. Se distingue con bastante nitidez, por ejemplo, de las opciones epistemológicas clásicas del fundamentalismo, del coherentismo y del escepticismo. Y también de otras posiciones más recientes.

Dediquemos unas líneas a estos contrastes. Y empecemos por el fundamentalismo. Puede considerarse que las virtudes epistémicas son el fundamento del conocimiento, pero tal fundamento sería muy diferente del ofrecido por el fundamentalismo clásico. Mientras que éste último es internista y evidencialista, las virtudes epistémicas sugieren otra cosa. Las disposiciones a conocer son rasgos de nuestra subjetividad inseparables del mundo externo, son rasgos sólo definibles a través de las conexiones entre lo interno y lo externo. Además, en contraste con las evidencias, las virtudes no necesitan ser epistémicamente accesibles para el sujeto que conoce. Un sujeto virtuoso no necesita saber que lo es. Aunque, por supuesto, saber que lo es pueda implicar una mayor virtud.

Una epistemología basada en la virtud también puede integrar abundantes ingredientes coherentistas, por ejemplo al hacer que las relaciones que las creencias mantienen entre sí, y las relaciones entre creencias y meta-creencias sobre esas creencias, puedan ser fuentes de justificación. Sin embargo, una epistemología basada en la virtud es hostil a la idea coherentista clásica de que lo único que puede justificar una creencia es otra creencia. Las virtudes no son creencias. Por lo tanto, si las virtudes epistémicas justifican nuestras creencias, lo que justifica una creencia no es, o en cualquier caso no es siempre, otra creencia.

Por lo que respecta al escepticismo, debemos comenzar señalando que aunque muchas veces se presente en combinación con el relativismo, tiene en sí mismo 
una identidad diferenciada, con lo cual el relativismo podría añadirse como una cuarta opción clásica. ${ }^{7}$ En cualquier caso, el énfasis epistemológico en la virtud insinúa otras posibilidades. Si el conocimiento depende de la virtud, si llegar a conocer plenamente depende de una serie de capacidades, competencias, habilidades, etc., que están a nuestro alcance, entonces el escepticismo en su sentido más radical, un escepticismo que afirme la imposibilidad del conocimiento, no tiene cabida. Y tampoco un relativismo radical que rechace la posibilidad de objetividad, racionalidad y progreso en el conocimiento. No tienen cabida, simplemente, porque no existen las "virtudes imposibles". Las virtudes epistémicas pueden ser muy costosas y difíciles de conseguir, pueden ser muy infrecuentes, etc., pero no pueden ser imposibles. Otra forma de expresar esta idea sería la siguiente. Puede incluso argumentarse que nunca llegaremos a entender completamente lo que es el conocimiento, o que no llegaremos a saber realmente lo que significa la palabra "conocer", pero si en algún sentido relevante poner en práctica ciertas virtudes epistémicas implica conocer, entonces el conocimiento ha de ser posible. ${ }^{8}$

La epistemología basada en la virtud también se distingue de la opción epistemológica, más reciente, del fiabilismo. ${ }^{9}$ Para el fiabilismo, los valores y normas epistémicamente relevantes, y la justificación, la racionalidad, y el propio conocimiento, se constituyen por su relación con la verdad. Una epistemología basada en la virtud sintoniza con este planteamiento. Puede aceptar, por ejemplo, que una creencia esté justificada al proceder de una fuente, o método, "veraz"10. Esto, sin duda, supone

\footnotetext{
${ }^{7}$ El relativismo implica una serie de afirmaciones positivas sobre la naturaleza del conocimiento, el escepticismo sólo implica afirmaciones negativas, afirmaciones sobre lo que el conocimiento "no es". El relativismo es más sustantivo que el escepticismo.

${ }^{8}$ Que no podamos llegar a saber con completa generalidad qué es el conocimiento, al menos desde los marcos epistemológicos heredados, y que por lo tanto sea imposible una teoría completamente general del conocimiento, son las tesis principales de la posición escéptica de Barry Stroud. Véase Stroud (1984 y 2002). Véase también la interesante polémica Sosa-Stroud recogida en Sosa y Stroud (1994).

${ }^{9}$ Empleamos el término "fiabilismo" para traducir el término inglés "reliabilism". Es frecuente encontrar también la traducción castellana “confiabilismo”. La mayor brevedad justifica nuestra elección.

10 También podríamos decir "fiable", o "confiable". Pero en este caso, el término castellano "veraz" refleja perfectamente el significado pretendido. Pueden encontrarse planteamientos fiabilistas en F. Ramsey, P. Unger y D. Armstrong. Más recientemente, tres importantes obras de referencia son Dretske (1981), Goldman (1986) y Nozick (1981). Para el primero, la noción clave es la de información; para el segundo, la de relaciones causales apropiadas entre los sujetos y sus entornos; para el tercero, la verdad de ciertos condicionales materiales subjuntivos.
} 
una virtud. Pero aún más virtuoso sería, cabe decir meta-virtuoso, saber que lo es. Y la epistemología basada en la virtud, sobre todo en la formulación de Sosa, tiene muy en cuenta estas posibilidades reflexivas. El fiabilismo puede asumirse. De algún modo, conocer ha de implicar que nuestras creencias son capaces de "rastrear la verdad" ("tracking the truth")". Pero muchas veces, sobre todo en sujetos epistémicos como nosotros, también debería exigirse que esa pista se siga de manera "reflexiva". El sujeto ha de poder conocer que se sigue la pista de la verdad. Con ello aumenta la virtud operante, o incluso puede cambiar cualitativamente. ${ }^{12}$

Hay otra opción epistemológica, aún más reciente que el fiabilismo, que debemos mencionar. Se trata de una importante cristalización de la vieja sospecha de que el concepto de conocimiento sea tan importante como no analizable mediante otros conceptos más básicos. ${ }^{13}$ A través del concepto de conocimiento podrían analizarse otros conceptos, pero él mismo sería un concepto máximamente primitivo, originario, básico. Y no sería un concepto eliminable, como podría llegar a serlo para el escepticismo, para el relativismo, o incluso para algunas variedades de coherentismo. Sería un concepto filosófico imprescindible. Y también podría ser un concepto psicológico imprescindible. Pero aún sin ser analizable, el concepto de conocimiento tendría implicaciones cruciales. ${ }^{14}$ Por ejemplo, que atribuir cono-

11 "Tracking the truth" es una expresión abundantemente usada en la literatura fiabilista. De acuerdo a Nozick (1981), conocer que $p$ (llamémoslo, $K p$ ) de alguna manera ha de entrañar estos dos condicionales: 1) Si $K p$, entonces $p$; y 2) Si $p$, entonces $K p$. Serían condicionales materiales subjuntivos, con una mayor o menor fuerza modal. El conocimiento puede "rastrear la verdad" en estos sentidos. Como indicábamos en la nota 4, para Sosa es muy importante diferenciar los dos condicionales. El primero definiría la "safety", el segundo la "sensitivity".

${ }^{12}$ Por supuesto, conocer que se sigue la pista de la verdad es, a su vez, seguir la pista de la verdad. Es seguir la pista de "otra" verdad. Y si esto último incluye elementos del primer "rastreo", lo cual parece inevitable, la empresa entrañará siempre un cierto tipo de circularidad epistémica.

${ }^{13}$ Como dijimos, esta opción epistemológica ha sido recientemente promovida por Williamson (2000). ¿Cómo se relacionan conceptualmente las tesis de Stroud, sobre la imposibilidad de conocer qué sea el conocimiento en general, con esta posición epistemológica que considera que el conocimiento es en último término indefinible? Buena pregunta.

${ }^{14}$ Esta posición guardaría también un estrecha similitud con la posición de Moore respecto al concepto de "lo bueno", también primitivo y no analizable. En este sentido, la analogía podría continuar relacionando el surgimiento de problemas tipo Gettier, respecto a todas las propuestas por considerar que el conocimiento puede definirse como un tipo de creencia verdadera y justificada de algún modo peculiar, con el argumento de la pregunta abierta de Moore ("open-question argument") acerca de la posibilidad de seguir siempre preguntando, en relación a cualquier propuesta que intentara definir lo bueno a través de otra serie de 
cimiento a un sujeto sea atribuirle un estado mental "factivo" (un estado mental que implica ciertos hechos extra-mentales, que implica que tienen lugar ciertos hechos en el mundo), realmente sería el estado mental máximamente factivo.

Atribuir conocimiento sería atribuir un estado mental que necesariamente tiene un contenido verdadero. $Y$ en lugar de entender el conocimiento como cierto tipo de creencia (creencia que es verdadera, que está justificada, etc.), este enfoque propone entender la creencia en términos de conocimiento. El estándar de la creencia, la norma de la creencia, sería el conocimiento. El horizonte del conocimiento ofrecería garantías a la creencia, y no al revés. Creer que $p$ es tratar a $p$ como si fuera conocimiento, o como pretendiendo serlo. El lema de esta concepción puede resumirse así: "El conocimiento es lo primero" (Knowledge First). ${ }^{15}$

Una epistemología basada en la virtud también ofrece alternativas a esta opción epistemológica. De acuerdo a Sosa, la noción de "experiencia apta" conseguiría los mismos beneficios teóricos que el considerar que el conocimiento es un concepto primitivo y no analizable. Y podría hacerlo sin tener que renunciar a entender qué es el conocimiento. ${ }^{16}$ La aptitud de la experiencia consistiría en su capacidad para manifestar un logro (por ejemplo, la formación de una creencia perceptiva) como consecuencia del ejercicio de una serie de capacidades (disposiciones, habilidades, virtudes, etc.) por parte del sujeto.

propiedades (en particular de tipo naturalista), si no es posible que algo no sea bueno aún teniendo esas propiedades, y si no es posible que algo sea bueno aún sin tener esas propiedades. ${ }^{15}$ Con todo, como hemos dicho, conocer puede perfectamente seguir implicando tener creencias verdaderas y justificadas (acaso con algún otro rasgo añadido). Sin embargo, esto no definiría qué es conocer. Conocer podría también implicar una estructura de tipo fundamentalista. Pero esto tampoco definiría lo que es el conocimiento. Si reconsideramos la última parte del Teeteto, de Platón, podría tal vez encontrarse un antecedente clásico de esta opción epistemológica. Una vez descartado que el conocimiento sea sensación, que sea opinión verdadera, y que sea opinión verdadera basada en razones (lo cual constituiría la concepción tripartita del conocimiento, campo sobre en el que se desarrolla toda la problemática ligada a los casos tipo Gettier), Platón deja abierta la posibilidad de que conocer sea el resultado de una intuición racional en la que se manifiesta una evidencia que no puede no ser cierta. El fundamentalismo consideraría esto como una opción epistemológica que nos permite entender qué es el conocimiento. Para la opción que estamos examinando, afirmar esta posibilidad sería equivalente a decir que el conocimiento es indefinible. Curiosamente, esta misma interpretación es adoptada por el escepticismo, académico y pirrónico, vinculado a Platón y también con las tendencias místicas del platonismo posterior. ${ }^{16}$ Sosa discute en detalle esta opción epistemológica (Knowledge First) en el capítulo cuatro de Sosa (2010). 


\section{VIRTUDES EPISTÉMICAS, CIRCULARIDAD Y PERSPECTIVA}

Las virtudes epistémicas presentan tres importantes rasgos. El primero de ellos es su fuerte carácter relacional. Las virtudes epistémicas son propiedades intensamente relacionales. Todas las disposiciones tienen una naturaleza relacional. Pero en el caso de las virtudes epistémicas este rasgo se acentúa. Como poco, necesitan definirse en relación a un entorno material y en relación a un contexto intencional ${ }^{17}$. Y también en relación a un cierto sujeto, o cuando menos en relación a un cierto "tipo" de sujeto. Indudablemente, todo esto también nos aleja del fundamentalismo y del coherentismo clásicos, por lo menos de sus versiones menos elaboradas. ${ }^{18}$

El segundo rasgo es la gradualidad. Las virtudes epistémicas suelen poseerse en mayor o menor medida. En cualquier caso, no parece que nosotros podamos tener ninguna virtud epistémica en un grado supremo. Esto se aplica inmediatamente a cosas como el tener evidencias, disponer de razones, poseer justificación, etc. Y conlleva una profunda reconsideración de la idea clásica de que el conocimiento ha de tener unos fundamentos absolutamente sólidos, más allá de cualquier posibilidad de revisión y mejora. Si el fundamento del conocimiento es la virtud, se tratará de un fundamento siempre revisable y siempre capaz de mejora. Es más, será también un fundamento muy sensible a los entornos reales y a los contextos intencionales de posesión, atribución y evaluación. ${ }^{19}$

${ }^{17}$ Cabría distinguir de esta forma los entornos de los contextos. Los entornos serían entidades materiales, los contextos entidades intencionales. El punto de vista adoptado a la hora de identificar unos y otros es diferente. Identificar un contexto requiere siempre una determinada "perspectiva intencional". Identificar un entorno no lo requiere, supuestamente.

${ }^{18}$ A menudo, Sosa describe su epistemología basada en la virtud como fruto de una revisión actualizada y elaborada del fundamentalismo, capaz de hacer frente a problemas cruciales como el de la "speckled hen" (véase la nota siguiente). Versiones actuales y elaboradas del coherentismo se encuentran en Laurence Bonjour, Donald Davidson, Keith Lehrer, y Nicholas Rescher. Véase Bonjour (1985), Davidson (1986), Lehrer (1986), y Rescher (1973). En Bonjour y Sosa (2003) se intenta ofrece un contraste ilustrativo de las diferencias entre una epistemología coherentista y una epistemología basada en la virtud.

${ }^{19}$ A pesar de ser un fundamentalista, Roderick Chisholm (1942) inicia una potente línea crítica frente al mismo diferente de la línea conceptualista abierta por Wilfrid Sellars. Las diferencias son muy importantes. El frente abierto por Chisholm podría seguir en pie aunque se rechazara el conceptualismo de Sellars. Puede concretarse en el problema de la "speckled hen". Se trata de lo siguiente. Estamos viendo una gallina moteada. Sus plumas tienen una serie de manchas identificables. Desde nuestra perspectiva, sus plumas deberían presentar un cierto número 
El tercer rasgo de las virtudes que quiero resaltar se deriva de la normatividad presente en toda disposición. Las disposiciones no son reducibles a regularidades, ni causales ni estadísticas. Son modos de ser llenos de poder contrafáctico, en particular de poder normativo. ${ }^{20}$ Una epistemología basada en la virtud permite así entender que las conexiones entre la epistemología y la ética sean tan estrechas. Y más aún, cuando esa epistemología y esa ética están a su vez basadas en la virtud. ${ }^{21}$ Para una ética basada en la virtud, la fuente última de los valores y normas morales también se situaría en ciertas disposiciones de los sujetos y de las comunidades morales. En ética, esto se opone a concepciones como las del fundamentalismo ético, propio del intuicionismo o del deontologismo; también se opone al mero coherentismo ofrecido por el contractualismo o el consensualismo; a las estrategias utilitaristas o pragmatistas que definen los valores y normas morales por su conexión con la utilidad o el éxito práctico; y a las posiciones radicales del escepticismo y relativis-

determinado de tales manchas. Ahora bien, ¿cuántas? Supongamos que nuestra visión de la gallina provoca en nosotros la creencia de que la gallina tiene 48 manchas. ¿Es suficiente nuestra visión directa de la gallina, el conocimiento por familiaridad ("by acquaintance") que tenemos de ella, para justificar esta creencia? La respuesta es claramente negativa. Y sin embargo, éste sería justo el tipo de conocimiento que el fundamentalismo clásico querría situar en la base, en los fundamentos, de todo nuestro conocimiento. Este problema aparece ya en Ryle. Incluso en el propio Descartes. Éste último, en su Meditación Sexta, usa el ejemplo de un chiliágono (un polígono de 1000 lados) para ilustrar la diferencia entre el entendimiento y la imaginación. Podemos imaginar un triángulo o un cuadrado, y podemos entender lo que son. Pero no podemos imaginar un chiliágono, o un miriágono (un polígono de 10.000 lados). Sólo podemos entender lo que son. Con todo esto, resulta muy problemático identificar el tipo de evidencias capaces de servir de fundamentos absolutamente sólidos del conocimiento. Sobre este problema, véase también Kennedy (1993), Markie (2009), y Poston (2007). Sosa lo ha tratado en diferentes lugares, insistiendo siempre en lo borrosa que es la separación entre imaginar, o percibir, por un lado, y entender, o concebir, por otro. ¿Podemos imaginar un polígono de 20 lados, o tan sólo entender qué es? ¿Y un polígono de 10 lados? El análisis del problema de la "speckled hen" es para Sosa una importante vía para introducir las nociones de "facultad cognitiva" y "virtud epistémica", a fin de preservar formalmente un cierto tipo de fundamentalismo. Nuestras facultades cognitivas, nuestras virtudes epistémicas, nos permiten formar creencias generalmente veraces, en base a nuestra experiencia, acerca de los lados que tienen los polígonos de, digamos, menos de 8 lados; pero no acerca de los lados que tienen los polígonos de 8 o más lados. El fundamento del conocimiento estaría constituido por un conjunto de tales "facultades virtuosas".

${ }^{20}$ Sobre este tema, véase Broncano y Vega (2009).

${ }^{21}$ Véase al respecto DePaul, M. y L. Zagzebski (eds.) (2003). 
mo moral. ${ }^{22}$ Sin embargo, también en el caso ético, como en el epistemológico, un enfoque basado en la noción de "virtud" (recordemos una vez más: disposiciones, capacidades, competencias, aptitudes, habilidades, carácter, etc.) es capaz de integrar abundantes elementos de todas las anteriores posiciones.

La noción de virtud epistémica nos conduce a perspectivas ampliamente integradoras. Y la epistemología de Sosa es un magnífico ejemplo de tal integración múltiple. En sus propuestas se asume la necesidad de un cierto fundamentalismo; ${ }^{23}$ también encontramos un cierto coherentismo complejo y muy abierto, en el que la experiencia aún puede desempeñar un papel importante; $y$ un fiabilismo que reconoce el protagonismo de la búsqueda de la verdad en nuestra actividad epistémica más básica. Así mismo, está presente un cierto relativismo en forma de relatividad conceptual. Y la discusión en detalle del problema del criterio y del escepticismo, sobre todo de la problemática pirrónica que encontramos en Descartes, conduce a la distinción crucial entre, por un lado, un tipo de "conocimiento animal", sumamente básico y con una dinámica fiabilista, y por otro lado un tipo de "conocimiento reflexivo" que intenta situar al anterior en una perspectiva epistemológica auto-comprensiva.

Acabamos de destacar varios rasgos importantes de las virtudes epistémicas. Antes hemos ofrecido un rápido bosquejo de la epistemología de Sosa basada en la virtud, comparándola con otras perspectivas epistemológicas. Digamos ahora algo sobre el aristotelismo manifiesto que acompaña tal enfoque.

${ }^{22}$ Los paralelismos de estas posiciones éticas con las posiciones epistemológicas aludidas unos párrafos atrás son obvios. Con todo, pocas veces se han señalado las conexiones del utilitarismo y del pragmatismo en ética con el fiabilismo en epistemología. En el primer caso, hay un "rastreo" de lo útil, o de lo que tiene éxito práctico. En el segundo caso, hay un "rastreo" de la verdad. Por supuesto, también hay un utilitarismo y un pragmatismo epistemológicos. Y en ellos, la verdad se sustituye por la utilidad o por el éxito en la acción.

${ }^{23}$ De un fundamentalismo de tipo formal. De acuerdo a Sosa, el "fundamentalismo formal" reconoce que el conocimiento ha de tener como fundamento un cierto tipo de justificación, y que esa justificación ha de estar determinada por, o ha de sobrevenir a, propiedades o relaciones no normativas ni evaluativas. El "fundamentalismo sustantivo" añadiría una propuesta concreta acerca de en qué puede consistir esa justificación, una propuesta que generalmente ha sido internista y evidencialista. Desde esta concepción, el coherentismo también sería una variedad de fundamentalismo formal. Y lo opuesto al fundamentalismo formal sería, simplemente, el "pesimismo epistemológico". Véase Sosa (1991:151). Esta distinción entre un fundamentalismo formal y una cierta variedad de fundamentalismos sustantivos (en teoría, una gran variedad; en la práctica, algo mucho más restringido) podría llevarse a cabo a propósito de cualquier propiedad normativa. 
Cabe identificar diversas concepciones de las virtudes. Cada una de ellas se vincularía a un diferente modelo. Podríamos, por ejemplo, distinguir entre:

- Modelo artesanal: Centrado en un "saber hacer bien algo", adquirido con trabajo y esfuerzo, casi siempre en el seno de una cierta comunidad, o subcomunidad, social muy específica que sirve de referencia a la hora de fijar los contextos adecuados de producción y evaluación.

- Modelo religioso: La virtud siempre es aquí un "don" muy especial, algo "dado" que requiere una "gracia" particular. Con mucha frecuencia, la virtud se conecta aquí también con la "sabiduría". Una sabiduría que llega más allá de donde puede llegar cualquier conocimiento o práctica particular.

- Modelo ético: En este modelo, cobran relieve pares combinados de nociones como las de "carácter" y "decisión", "responsabilidad" y "culpa". A veces pueden tener más peso unas nociones, otras veces otras.

- Modelo político: La virtud es aquí el "instrumento" o el "medio" para conseguir una serie de fines. Las nociones importantes son las de "estrategia", "negociación", "equilibrio" y "prudencia".

- Modelo deportivo: La virtud tiene aquí una fuente natural e innata, y también un desarrollo propio que, en un contexto benigno, si no hay impedimentos que interfieran, conduce a ciertos logros característicos que pueden llegar a medirse a través de éxitos evaluables en situaciones competitivas.

Seguramente haya más modelos, pero estos son importantes. En Platón está muy presente tanto el modelo artesanal como el modelo religioso (lo cual, por cierto, entraña un importante problema interpretativo). En Aristóteles, cobra relieve el modelo ético y el modelo político (que también pueden llegar a mantener entre sí relaciones muy tensas). Éste último modelo, el político, es llevado a cierta posición extrema por Maquiavelo. Por su parte, el modelo deportivo está muy presente en Nietzsche.

Al lado de otros modelos (sobre todo del modelo artesanal, del modelo ético y del modelo político), este modelo deportivo también está muy presente en Sosa. ${ }^{24} \mathrm{Y}$ su principal mérito es la simplicidad. Pero es obvio que puede llegar a dar problemas. Sobre todo cuando nos planteamos la cuestión de la "unidad de la virtud". Y también cuando nos enfrentamos a la evaluación de los logros de nuestras actuaciones fuera de ámbitos de competición claramente definidos ${ }^{25}$.

${ }^{24}$ Véase, sobre todo, Sosa (2010 y 2015). Más adelante nos detendremos en este punto.

${ }^{25}$ El tema de la "unidad de la virtud" es fuertemente aristotélico. Las diferentes virtudes 
Como puede apreciarse, las diferencias entre todos estos distintos modelos pueden ser muy relevantes. Pero no nos detendremos a analizarlos en detalle. Sólo insistiremos en ciertos elementos aristotélicos que acompañan a la mayoría de los planteamientos epistemológicos actuales basados en la virtud.

Platón concebía la virtud como requiriendo siempre algún tipo de conocimiento máximamente cualificado. Ya hemos indicado que en parte (aunque sólo en parte) su concepción de la virtud sigue el modelo religioso. No hay virtud sin sabiduría. Esto se aplica de un modo muy especial a las virtudes epistémicas. Las virtudes epistémicas requieren un conocimiento máximamente cualificado, que para Platón acaba siendo siempre un conocimiento innato.

Aristóteles concebía la virtud de un modo mucho menos intelectualista. Y por ello, mucho más generalizable a ámbitos tanto humanos como no humanos. Las virtudes son potencialidades. Un tipo peculiar de potencialidad que se manifiesta y modula a través de sus manifestaciones. Cada sustancia (especialmente plantas y animales, pero muy especialmente las personas) posee un conjunto característico de potencialidades. En las circunstancias y contextos adecuados, ciertas actividades conducen a la plena realización de esas potencialidades que les son propias. Y en el caso especial humano, esa plena realización constituye importantes excelencias: la felicidad moral, el logro epistémica, el éxito práctico, etc.

Este aristotelismo está muy presente en la filosofía actual. Y los actuales enfoques epistemológicos basados en la virtud siguen la orientación aristotélica. ${ }^{26}$

La reivindicación de las disposiciones como constituyentes no prescindibles de la realidad, así como la reivindicación del valor explicativo irreducible del propio

de un sujeto deben tener una estructura orgánica, deben estar organizadas, unificadas, de acuerdo al fin último relevante. En el caso de los seres humanos, ese fin último es la felicidad. Siguiendo la dialéctica de Sosa (2010), la pregunta pertinente sería: ¿Cómo la aptitud y la metaaptitud epistémicas se conectan con nuestra felicidad? Y en este punto, la comparación con el deportista de competición o con Diana la Cazadora fácilmente se quiebra, y deja de ayudarnos en nuestros análisis. Pues, ¿cómo la aptitud y meta-aptitud del deportista de competición o de Diana la Cazadora contribuyen a su felicidad? ¿Qué formas de hacerlo son adecuadas y cuáles no? Es obvio que estas cuestiones sobre la "unidad de la virtud" han de tener implicaciones importantes respecto a la distinción entre un "conocimiento animal" y un "conocimiento reflexivo", y su adecuada integración en el caso humano.

${ }^{26}$ Sobre los diferentes enfoque que puede llegar a adoptar una epistemología basada en la virtud, véase Alfano (ed.)(2015), Axtell (1997), DePaul y Zaggzebski (eds.)(2003), Greco (1992, 1993 y 2002), Greco y Turri (eds.)(2012), Kvanvig (1992) y Zagzebski (1996). 
lenguaje disposicional, es un aspecto muy importante de tal aristotelismo. ${ }^{27}$ Otro aspecto no menos importante se vincula al reconocimiento del papel crucial que desempeña la reflexión, y la búsqueda prudente de equilibrios, en todos los fenómenos normativos propiamente humanos. La búsqueda reflexiva de equilibrios es crucial en ámbitos como la ética, la política o la epistemología. ${ }^{28}$

Para Aristóteles, la virtud se sitúa siempre entre determinados extremos. En este componente del aristotelismo se escuchan ecos platónicos. Es más virtuosa una virtud acompañada de reflexión equilibrada que una virtud irreflexiva. Y sin embargo, la búsqueda prudente y reflexiva de equilibrios es ya algo muy distinto del auténtico conocimiento platónico, basado en el esfuerzo dialéctico por hacer explícito un conocimiento implícito, que ya ha estado siempre con nosotros, llegando a tener una incuestionable intuición racional o visión intelectual.

Todo esto también se encuentra en la obra de Ernesto Sosa. Las nociones disposicionales acaban resultando indispensables a la hora de adoptar una perspectiva epistemológica adecuada. Y la búsqueda de equilibrios reflexivos en sentido amplio es un elemento crucial en su enfoque. Es más, la perspectiva reflexiva que ofrece tal búsqueda se convierte en la estrategia fundamental con la que hacer frente a los problemas de circularidad. Simplemente, la reflexión convierte algunas circularidades en fuentes de conocimiento.

La mayoría de las ideas que configuran la epistemología basada en la virtud ofrecida por Sosa se perfilan ya en su libro, de 1991, Knowledge in Perspective, Selected Essays in Epistemology. ${ }^{29}$ En este libro podemos encontrar planteamientos que son cruciales para entender el desarrollo de los planteamientos de Sosa. Destacan cuatro ideas:

1) La inevitabilidad de equilibrios reflexivos entre la constatación de hechos epistémicos y el establecimiento de criterios normativos con los que identificar y enjuiciar tales hechos, lo cual es relevante tanto a la hora de entender el análisis conceptual como los proyectos de naturalización (dos de las tradiciones más influyentes en la filosofía de las últimas décadas), y conduce obviamente a situaciones de circularidad.

\footnotetext{
27 Son muchos los nombres propios que protagonizan la actual reivindicación de las disposiciones: A. Bird, T. Crane, B. Ellis, J. Heil, C. B. Martin, D. Mellor, G. Molnar, S. Mumford, U. T. Place, H. Sankey, etc. Sobre la conexiones de tal reivindicación con lo que se ha llamado "nuevo aristotelismo" en otros campos, véase Groff y Greco, (eds.)(2013).

${ }^{28}$ En el campo de la ética, han sido muy influyentes los planteamientos de A. McIntyre y de M. Nussbaum. Véase, por ejemplo, McIntyre (1981) y Nussbaum (1986).

${ }^{29}$ Sosa (1991). En conexión con esta obra de Sosa, véase Liz (2003).
} 
2) La necesidad de apelar en los análisis epistemológicos a la noción de facultades virtuosas y, en general, de virtudes epistémicas. En ambos casos, se trataría de determinadas disposiciones a tener creencias de un modo tal que se eleve su estatus epistémico, pasando a constituir distintas clases de conocimiento en diverso grado. Esas disposiciones pueden ser más o menos innatas o adquiridas. Y también puede haber una mayor o menos contribución de los entornos reales y de los contextos intencionales de las comunidades epistémicas. Una facultad virtuosa podría fácilmente definirse como una facultad capaz de producir resultados epistémicamente virtuosos.

3) La necesidad de reconocer componentes externistas y sociales en el conocimiento. Lo que es virtuoso es relación a un cierto entorno real puede dejar de serlo en relación a otro entorno. Y lo no virtuoso también puede pasar a serlo con un cambio de entorno. De modo similar, lo virtuoso y lo no virtuoso dependen de los contextos sociales.

4) La existencia, en sujetos como nosotros, de dos niveles epistémicos en interacción: un nivel de conocimiento animal, constituido por virtudes epistémicas que arrojan un cierto resultado sin que este proceso tenga que ser epistémicamente accesible al sujeto, y menos aún conocido con justificación, y un nivel de conocimiento reflexivo en el que se intenta conocer nuestra posición epistémica en el mundo usando los materiales ofrecidos por el conocimiento animal y otros materiales exclusivos de la propia actividad reflexiva.

Muchos de estos temas son retomados con fuerza en los dos volúmenes de Sosa titulados Apt Belief and Reflective Knowledge. ${ }^{30}$ Sobre todo, se utiliza en diversos frentes la distinción establecida con anterioridad entre conocimiento animal y conocimiento reflexivo. El tipo de logro epistémico que implican es muy diferente. En el primer caso, el objetivo de la creencia es la verdad. Ser sensibles a la verdad y estar seguros de ella en nuestras creencias. ${ }^{31}$ En el segundo caso, el objetivo es la explicación comprehensiva (o la comprensión explicativa) de nuestra posición epistémica en el mundo.

\footnotetext{
${ }^{30}$ Sosa (2007) y Sosa (2009). Estos dos volúmenes aparecerán próximamente traducidos al castellano en la misma editorial en la que se ha traducido ya Sosa (2010).

${ }^{31}$ Como hemos dicho, podemos representar la sensibilidad a la verdad mediante el condicional subjuntivo "Si fuera el caso que $p$, entonces se creería que $p$ ” y, a su vez, la seguridad de la creencia mediante el condicional subjuntivo "Si se creyera que p, entonces sería el caso que p". Nozick (1981) inicia ésta versión del fiabilismo. Y Sosa hace muchas veces uso de estos conceptos al analizar las nociones de virtud epistémica y de facultad virtuosa. Sobre las relaciones entre creencia y verdad, véase también Engel (2007).
} 
El segundo de estos volúmenes se centra en el problema de la circularidad epistémica. Se subtitula Epistemic Circularity. Trata sobre las circularidades que se producen cuando al intentar validar reflexivamente nuestro conocimiento, nos vemos una y otra vez llevados tener que usar ese mismo conocimiento. Merecerá la pena examinar brevemente algunas de sus principales tesis.

La importancia del problema de la circularidad se ilustra, en la Parte I del libro, a través de los planeamientos de autores como Moore, Reid, Descartes, Hume, Wittgenstein, Strawson, Davidson o Sellars. Tal circularidad parece inevitable. Más pronto o más tarde, justificar que conocemos implicará asumir que ya conocemos. El escepticismo, y otras posiciones filosóficas, han utilizado muchas veces a su favor este fenómeno. El tratamiento que Sosa ofrece es muy diferente. Según Sosa, la circularidad epistémica no siempre es viciosa. En la Parte II del libro, se desarrolla una concepción epistemológica en la que las circularidades virtuosas tienen un destacado papel. Esa concepción vuelve a ser una epistemología basada en la virtud. Y lo que Sosa defiende es que, en ciertas condiciones, la circularidad misma es una virtud. Dicho de otro modo, en ciertas condiciones la circularidad también puede ser una importante fuente de conocimiento.

En el capítulo 1 del libro, titulado La prueba de Moore, Sosa analiza el famoso argumento de Moore acerca de las propias manos como objetos de un mundo externo que conocemos básicamente a través de la percepción y de la memoria. La prueba de Moore se dirige más contra el idealismo que contra el escepticismo. Y Moore dedica la mayor parte de sus esfuerzos a analizar la noción de mundo externo. Pero la cuestión del escepticismo está también presente, sobre todo en el contexto de otros trabajos de Moore durante esos años. Para rechazar el escepticismo sobre la posibilidad de conocer el mundo externo, deberían asegurarse cosas como que no estemos soñando. $\mathrm{Y}$ es aquí donde aparece el problema del círculo. A diferencia de Russell, cuando se enfrenta a este mismo problema, Moore es fundamentalista. Sostiene que, aún sin ser capaces de ofrecer pruebas, sí podemos tener razones concluyentes capaces de apoyar nuestra creencia de no estar soñando. Aún sin ser capaces de ofrecer pruebas plenamente articuladas, cabe tener la certeza de poder conocer el mundo externo. El problema es cómo tener esas razones y esa certeza sin apoyarnos, sin presuponer, que conocemos ya cosas del mundo externo. Sosa ofrece en este punto el tipo de respuesta que desarrollará con detalle en la segunda parte del libro.

Seguramente, tener razones concluyentes y certezas sea excesivo. Pero sí existen fuertes razones basadas en la reflexión. Para llegar a ellas, el primer paso sería distinguir conocimiento animal de conocimiento reflexivo. El primero no requeri- 
ría conocer que no estemos soñando, sólo requeriría que de hecho no estuviéramos haciéndolo. El segundo, el conocimiento reflexivo, sí requiere conocer que no estemos soñando. Sin embargo, no se exige que para conocer que nuestras manos están delante nuestro, tengamos que conocer de antemano que no estamos soñando. El conocimiento acerca de no estar soñando no tiene porqué ser un conocimiento previo, anterior a nuestro conocimiento del mundo externo. Basta con que sea un conocimiento independiente de cualquier presunto conocimiento particular del mundo externo. Y esto puede obtenerse a través de la reflexión.

El modelo para nuestras creencias sería el de una red. La red se conecta causalmente con el mundo sobre todo a través de la percepción y la memoria. Pero cada nudo de la red, cada creencia, recibe en mayor o menor medida el apoyo de todos los demás nudos. La creencia de no estar meramente soñando que percibimos un mundo externo, creer que realmente lo estamos percibiendo, puede así recibir el apoyo justificatorio de otras muchas creencias, apoyo que cabe detectar reflexivamente, sin depender de ninguna de ellas en particular. ${ }^{32}$

El caso, según Sosa, sería muy parecido al de conocer la velocidad de nuestro coche a través de un velocimetro. ¿Cómo saber si el velocímetro funciona correctamente? Utilizar para ello exclusivamente el propio velocímetro sería sin duda "viciosamente" circular. Sin embargo, entre los muchos conocimientos sobre los que nos apoyamos a la hora de evaluar el funcionamiento correcto de un cierto velocímetro, también ha de haber conocimientos que proceden de ese propio velocímetro. Esto conlleva circularidad. Pero no hay aquí ninguna circularidad viciosa si tal apoyo es suficientemente independiente de la información que obtenemos de ese velocímetro en particular.

La misma situación se presenta cuando evaluamos patrones de medida. Tal vez haya habido variaciones en los objetos particulares que tomamos como el "metro patrón”, por ejemplo. Para evaluar esto, tenemos que acabar midiendo longitudes. Y esto encierra circularidad. Pero no es una circularidad viciosa si nuestra medición es suficientemente independiente de los objetos particulares que estamos evaluando. Y lo mismo ocurriría cuando nuestros patrones no son objetos particula-

\footnotetext{
${ }^{32}$ Este modelo de la justificación como " $r$ d", en contraste con una justificación transmitida linealmente a través de una "tubería" es desarrollado por Sosa en el último de los capítulos del libro. Es un buen modelo. Lo que estoy sugiriendo en el presente trabajo es que tal red se extiende por toda nuestra comunidad epistémica. Y que también se extiende temporalmente hacia el futuro.
} 
res sino entidades teóricas (por ejemplo, la velocidad de la luz). Las circularidades son inevitables. Pero no toda circularidad es viciosa. Hay circularidades virtuosas.

La interpretación que Sosa ofrece de Descartes, muy especialmente de los llamados "círculos cartesianos", en diversas partes de este libro, y en otros trabajos, es sumamente novedosa y sugerente. Tradicionalmente, Descartes ha sido considerado uno de los máximos exponentes de una epistemología fundamentalista de tipo evidencialista. Bajo la interpretación de Sosa, Descartes se convierte en paradigma de un fiabilismo que busca en cierto tipo de circularidad virtuosa un incremento de la justificación.

De acuerdo a Sosa, la distinción que Descartes establece en sus textos entre "cognitio" y "scientia" sería análoga a su distinción entre conocimiento animal y conocimiento reflexivo. La cognitio permitiría un conocimiento fundamental que no precisa inferencias ni reflexión, que no requiere conocer que se conoce. La percepción, la memoria, etc., incluso la intuición, ofrecen esta variedad de conocimiento. Usando un conocido ejemplo de Descartes, podemos decir que este conocimiento es el conocimiento que se encuentra también al alcance del matemático ateo. Pero la cognitio no es el conocimiento más elevado que podemos tener.

El auténtico conocimiento sólo lo proporciona la scientia. Un matemático ateo no puede excluir que no esté siendo engañado en materias que a él le parecen evidentes. Y lo mismo podemos decir de un sujeto que percibe, que recuerda, etc. Sólo con cognitio, aunque su percepción no le engañe, aunque sus recuerdos sean fidelignos, no podemos descartar posibilidades como la del genio maligno. Esto sólo puede hacerlo un cierto tipo de reflexión, un cierto tipo de scientia. Como sabemos, Descartes busca esta scientia en la constatación de la existencia de un Dios veraz. Sus pruebas de la existencia de tal Dios, junto con otros elementos, van aportando los materiales con los que se construye una imagen muy coherente $y$ rica en contenido del mundo y de nuestra posición epistémica en él. Y esto va elevando el estatus de la cognitio hasta convertirla en scientia, en auténtico conocimiento. Para Descartes, este auténtico conocimiento implica certeza. Pero este ingrediente del planteamiento de Descartes, enfatizado por una larga tradición interpretativa, tal vez sea aquí el menos importante.

La scientia utiliza lo aportado por la cognitio. En las pruebas sobre la existencia de Dios, por ejemplo, se apela justamente al tipo de evidencia que puede llegar a considerarse problemática. Se apela a la intuición. Y también intervienen percepciones, recuerdos, etc. Hay ciertamente un círculo. Ahora bien, ¿se trata de un círculo que podamos llamar "vicioso"? Tal vez no. En cualquier caso, Sosa lo rechaza.

La misma estructura circular que encontramos en Descartes puede encontrarse también en otros muchos autores: Moore, Reid, Davidson etc. Los análisis de 
Sosa son aquí del máximo interés. El Descartes fundamentalista de la tradición, un Descartes sin relieve en busca de la certeza, adquiere ahora matices sorprendentes. Respecto a la cognitio, Descartes es claramente un fiabilista. Reflexivamente, intenta obtener scientia. Lo hace de una manera-sobrenaturalista. Y el resultado pretende tener certeza absoluta. Las estrategias de otros autores son diferentes. Pero lo que siempre se repite es la idea de que a través de la reflexión podemos construir una imagen coherente y rica en contenido de nuestra posición epistémica en el mundo capaz de elevar el estatus epistémico del conocimiento animal, el estatus epistémico de cualquier cognitio.

En el capítulo 8 del libro sobre la circularidad que estamos comentando, último de su Parte I, capítulo titulado Epistemology Naturalized, Davidson's Way, Sosa compara a Davidson con Descartes. Davidson intenta mostrar cómo la manera en que aprendemos los significados de nuestros lenguajes obliga a considerar que una gran parte de nuestras creencias, tal como pueden ser expresadas y descritas en esos lenguajes, han de ser verdaderas. Sosa considera que el papel desempeñado por estos argumentos de Davidson es similar al papel que desempeña en Descartes la apelación a un Dios benévolo respecto a nuestra actividad epistémica.

Examinemos ahora brevemente el último capítulo del libro, el 10, el último de la Parte II, más sistemática. Su título es Easy Knowledge and the Criterion. El llamado "problema del conocimiento fácil" ha irrumpido con fuerza en la epistemología de los últimos años. Podemos hacer una presentación general muy directa. Si para que nuestras creencias constituyan conocimiento necesitamos conocer que realmente constituyen conocimiento, entonces parece que nos vemos envueltos en regresos o círculos indeseables. Por lo tanto, si el conocimiento es posible, ha de haber creencias que constituyan conocimientos sin que debamos conocer que realmente constituyen conocimiento. Hasta aquí, no hemos salido de la tradicional problemática pirrónica ${ }^{33}$. Pero las cosas se ponen aun más feas cuando caemos en la cuenta de que si admitimos que ciertas fuentes son capaces de ofrecernos determinados conocimientos básicos sin que tengamos que conocer que lo hagan, entonces esos datos pueden ser utilizados en argumentos muy simples para llegar a conclusiones que han de parecernos muy difíciles de aceptar sin alguna justificación adicional. En otras palabras, parece obtenerse demasiado fácilmente un conocimiento que habitualmente consideraríamos muy difícil de justificar. Y de ahí, el propio nombre del problema.

${ }^{33}$ Véase al respecto Chisholm (1982 y 1989). 
Si asumo que la percepción me ofrece ciertos conocimientos básicos en un determinado caso particular sobre, por ejemplo, que delante de mí haya una pared roja, entonces como eso es incompatible con que realmente no haya tal pared roja delante de mí, sino tan sólo, por ejemplo, una pared blanca que por una especial iluminación me parece roja, debería concluir que conozco que delante mío no hay tal pared blanca extrañamente iluminada ${ }^{34}$. $Y$ eso que conozco me permitiría reafirmar que realmente conozco perceptivamente que frente a mí hay una pared roja. Así mismo, empleando una especie de argumento inductivo "autosustentador" 35, la acumulación de conocimientos básicos de este tipo procedentes de una cierta fuente me permitiría concluir que, en general, esa fuente es veraz. Si la percepción me ofrece conocimientos básicos, puedo concluir en base a lo que percibo que la percepción misma es veraz. Y lo mismo cabe decir de todas las demás fuentes de conocimiento

En todos estos casos estaríamos ante circularidades epistémicas. Lo que procede de una cierta fuente de conocimiento se utiliza para justificar que esa fuente es veraz, en un caso particular o en general. En apariencia, estas circularidades son viciosas. Parecen lo mismo que comprobar que el velocímetro de un coche funciona bien sólo mediante lecturas de ese velocímetro. El análisis que Sosa hace del problema del conocimiento fácil es tremendamente lúcido. El punto crucial está en el "sólo"de la última frase. Pues muchas de las formas que existen para comprobar si el velocímetro de un coche funciona bien incluyen sin ningún problema hacer lecturas de dicho velocímetro. Lo que ocurre es que también involucran muchas más cosas.

${ }^{34}$ Ésta es una versión muy común del problema del conocimiento fácil. Hace uso de cierto principio de exclusión bajo la implicación conocida que afirmaría que conozco que no se da todo aquello que conozco es incompatible con lo que conozco.

${ }^{35}$ El término técnico inglés utilizado en la literatura es "bootstrapping”. Otra de las versiones más comunes del problema del conocimiento fácil (véase la nota anterior) involucra esta estrategia. El término "bootstrapping” alude a la posibilidad de elevarse del suelo tirando hacia arriba de los cordones de nuestras botas o zapatos. También suele emplearse la figura de alguien elevándose del suelo tirando hacia arriba de su cabello. Un "proceso inductivo autosustentado" tomaría como premisas datos procedentes de cierta fuente en la que confiamos, y que de hecho es veraz sin que sepamos que lo es, llegando a la conclusión de que realmente es una fuente digna de confianza por ser veraz. Creo que cabría analizar estas "inducciones autosustentadoras" como cierto tipo de "inferencias de la mejor explicación" (o "abducciones"). La hipótesis que mejor explicaría los datos que tenemos, sobre todo si proceden de una fuente en la que confiamos, de una fuente que por otra parte podemos suponer que de hecho es veraz aunque no lo sepamos, es que su fuente realmente sea digna de confianza por ser veraz. 
Cuando las exclusiones de hechos que sabemos son incompatibles con nuestros conocimientos se hacen en un contexto reflexivo suficientemente coherente y comprensivo, pueden resultar muy plausibles. Y exactamente lo mismo cabe decir de las inducciones "autosustentadoras". Cuando se llevan a cabo en contextos adecuados, lo que considerado aisladamente parecía una circularidad viciosa puede convertirse en una circularidad de lo más virtuosa.

Sosa nos recuerda que, al fin y al cabo, los psicólogos y neurólogos que estudian los procesos perceptivos también se basan en lo que perciben (en lo que perciben en sus laboratorios, en lo que perciben en una pantalla de ordenador, en lo que perciben leyendo un artículo científico, etc.) para desarrollar sus propias teorías sobre la percepción. Y no pensamos que se encuentren en una situación epistémica de circularidad viciosa. Una manera arriesgadamente simple, pero con todo muy gráfica, de expresar esta idea sería diciendo que cuantas más premisas sea relevante añadir a un argumento viciosamente circular, a fin de poder llegar a su conclusión, más perderá ese argumento circular su carácter vicioso (más virtuoso llegará a ser).

Concluir $p$ simplemente suponiendo $p$ es ciertamente circular y vicioso. Concluir $p$ necesitando un largo argumento con premisas muy plausiblemente verdaderas, incluyéndose en algún momento $p$ como suposición, o acaso algo muy cercano a $p$, sigue siendo circular. Pero podría no ser ya un argumento tan vicioso.

De acuerdo a Sosa, el problema del conocimiento fácil apunta a algo epistemológicamente muy importante. Pero no apunta exactamente en la dirección que otros autores señalan. La solución al problema del conocimiento fácil no debe ser restrictiva sino ampliativa. No se deben limitar los principios epistémicos operativos (el principio de exclusión bajo la implicación conocida, la posibilidad de inducciones "autosustentadores", etc.). Más bien debemos considerar que la circularidad, en determinadas condiciones, es una fuente legitima de conocimiento.

Es más, debemos considerar que, en cuanto nos despegamos del conocimiento animal y nos adentramos en la aventura del conocimiento reflexivo, el tipo de conocimiento característicamente humano que ha sido crucial en la constitución de los seres que somos, inevitablemente la circularidad ha de ser una fuente legítima de conocimiento. Toda nuestra ciencia procede, en gran medida, de circularidades epistémicas. Y también, por supuesto, toda nuestra filosofía.

A través de las páginas de este libro que estamos comentando, vemos también que no puede combatirse al escepticismo sin incurrir en ciertas circularidades. Así mismo, que una filosofía basada en el análisis conceptual necesariamente debe asumir circularidades. Y que tampoco puede argumentarse a favor de perspectivas más naturalizadoras de los problemas filosóficos sin circularidad. Que cualquier 
intento por defender el valor de la verdad, de la objetividad, de la racionalidad, etc., será igualmente circular. Pero también, que no toda circularidad es epistemológicamente indeseable. Que hay circularidades viciosas, pero que también hay circularidades virtuosas.

\section{EL CONOCIMIENTO REFLEXIVO NOS INCLINA HACIA LA NO CRITERIALIDAD}

El conocimiento reflexivo requiere ascensos epistémicos. Y estos ascensos involucran perspectivas epistémicas amplias y la integración de circularidades epistémicas aceptables. ¿Qué virtudes epistémicas pueden estar aquí involucradas?

Mi hipótesis es que se necesita introducir una justificación basada en el buen ejercicio de ciertas capacidades para delegar y para aplazar la justificación. Para que las circularidades sean aceptables, han de acabar involucrando a otros sujetos de nuestra comunidad epistémica y también el desarrollo futuro de las actividades epistémicas que estemos llevando a cabo.

De una parte, comprobar que el velocímetro de un coche indica correctamente la velocidad del coche simplemente haciendo una lectura de dicho velocímetro es viciosamente circular. De otra, parece inevitable consultar tal velocímetro, o tal vez algo que suponemos puede ofrecer equivalencias relevantes a lo indicado por ese velocímetro, junto con muchas más cosas, a la hora de cualquier comprobación de su adecuado funcionamiento. Y aquí la circularidad no sería ya viciosa. De hecho, la mayoría de las formas que existen para comprobar si el velocímetro de un coche funciona correctamente incluyen hacer lecturas de ese velocímetro.

Supongamos ahora la siguiente situación epistémica, por lo demás muy común. Tengo plena confianz̧a, o algo muy cercano a ello, en que el velocímetro de mi coche marca correctamente la velocidad del coche. Podría equivocarme, pero mi confianza es máxima. Y puedo decir que sé que lo conoz̧co. ¿Cómo lo sé? ¿Cómo puedo tener yo ese conocimiento reflexivo?

No puedo ofrecer yo mismo las justificaciones pertinentes. Seguramente podría hacerlo si me pusiera a ello. Pero aquí y ahora yo no podría ofrecer tales justificaciones. Sin embargo, reflexivamente puedo afirmar que sé que conozco el velocímetro de mi coche marca correctamente la velocidad del coche. Sé que conozco eso. ¿En qué pueden basarse estos conocimientos reflexivos, que como he indicado serían sumamente comunes?

Mi respuesta es la siguiente. Esos conocimientos reflexivos se basan en mi capacidad para delegar y aplazar la construcción de las amplias circularidades inte- 
gradoras que justificarían explícitamente los conocimientos de primer orden implicados. Si nada me hace sospechar de lo contrario, confio en esas capacidades.

Tengo esos conocimientos reflexivos. Aunque siempre pueda equivocarme, en muchas circunstancias no puedo dudar de que sé que conozco que el velocímetro de mi coche marca correctamente la velocidad del coche. Mi confianza es máxima. $\mathrm{Y}$ tengo dicho conocimiento reflexivo en la medida en que funcionan bien mis capacidades para delegar y aplazar una justificación que involucraría amplias circularidades. Al funcionar bien, el ejercicio de esas capacidades se convierte en un ejercicio virtuoso. Y las propias capacidades para delegar y para aplazar se convierten en virtudes epistémicas reflexivas.

En general, disponer circularidades aceptables dentro de perspectivas epistémicas globales, de circularidades que puedan ser fuentes de conocimiento a medida que son cada vez. más amplias e integradoras, requiere la intervención en nuestro conocimiento reflexivo de ciertas capacidades para delegar y aplazar la justificación en el sentido siguiente:

1. Conocimiento reflexivo basado en una delegación de la justificación = Conocimiento reflexivo en base a justificaciones que no tienen porqué ser poseídas por el mismo sujeto que sea portador de ese conocimiento.

El conocimiento reflexivo ha de poder involucrar justificaciones delegadas capaces de hacer que sean correctas descripciones con la siguiente forma: - $x$ sabe que conoce que $p$ porque $y$ puede justificar ese conocimiento de que $p$ (siendo $x$ e $y$ sujetos diferentes).

2. Conocimiento reflexivo basado en un aplazamiento de la justificación = Conocimiento reflexivo en base a justificaciones que no tienen porqué existir de manera previa o simultánea a la posesión de ese conocimiento.

El conocimiento reflexivo ha de poder involucrar justificaciones aplazadas capaces de hacer que sean correctas descripciones con la siguiente forma:

- $x$ sabe en un tiempo $t$ que conoce que $p$ porque se podría justificar ese conocimiento de que $p$ en un tiempo $t^{\prime}$ (siendo $t^{\prime}$ posterior a $t$ ).

En 1, justifico mi conocimiento reflexivo delegando en otros sujetos. En 2, justifico el conocimiento reflexivo que tengo en cierto momento mediante un aplazamiento temporal.

En 1 hay algo más que la suposición de un posible conocimiento por testimonio. $\mathrm{Y}$ en 2 hay algo más que convicción subjetiva que acompaña a un conocimiento de primer orden que es posteriormente justificado. Ese algo más es un conocimiento reflexivo que se adquiere y mantiene gracias al ejercicio potencialmente virtuoso de ciertas capaci- 
dades para delegar y aplazar. Se delega o aplaza la justificación de cierto conocimiento que se considera reflexivamente. $\mathrm{Y}$ el delegar o aplazar de un modo virtuoso esa justificación que podemos llamar de primer orden nos hace estar justificados en nuestro conocimiento reflexivo.

Es importante distinguir el conocimiento por testimonio del conocimiento que delega la justificación. En el conocimiento por testimonio, el testimonio es una fuente de conocimiento. En el conocimiento que delega la justificación, la delegación es una fuente de justificación. Los papeles respectivos son muy diferentes. Podemos tener un conocimiento por testimonio que no delega la justificación y un conocimiento por testimonio que sí lo hace. En cualquier caso, el volumen y el alcance de la delegación puede ser mayor o menor. Y la delegación puede ser más o menos adecuada.

En el conocimiento que aplaza la justificación, tal aplazamiento también es una fuente de justificación. Nuevamente, el volumen y el alcance del aplazamiento puede ser mayor o menor. Y el aplazamiento puede ser más o menos adecuado.

También es importante apreciar que tanto en el conocimiento que delega la justificación como en el conocimiento que la aplaza hay siempre dos tipos de justificación involucradas. Una justificación de primer orden que se delega o aplaza. Y una justificación reflexiva que se obtiene mediante el ejercicio virtuoso de esas capacidades para delegar y aplazar.

Con estos ingredientes, cabe definir una variedad interesante de conocimiento. Podemos llamarla "conocimiento a crédito". Introduciremos primero la noción de crédito epistémico. La definición de conocimiento a crédito se hará directamente a continuación:

- Crédito epistémico = La condición epistémica que permite obtener un conocimiento mediante el ejercicio virtuoso de las capacidades para delegar o aplazar la justificación.

- Conocimiento a crédito = Creencia verdadera que puede ser vista reflexivamente como estando basada en un crédito epistémico. ${ }^{36}$

\footnotetext{
36 Suponemos que el ejercicio virtuoso de las capacidades para delegar o aplazar una justificación requiere un mínimo de espontaneidad epistémica. Tanto las virtudes morales como las virtudes epistémicas requieren un mínimo de espontaneidad. Es ambos casos, las capacidades o disposiciones se convierten en virtudes a través de alguna dosis de espontaneidad. Sin espontaneidad no hay virtud. Sin espontaneidad sólo hay "tendencia". No incluimos explícitamente este componente en nuestras definiciones.
} 
Muchos de nuestros conocimientos de primer orden son conocimientos a crédito. Y todo nuestro conocimiento reflexivo, cuando es suficientemente amplio, especialmente cuando quiere integrar circularidades virtuosas "autosustentadoras", acaba siendo conocimiento a crédito.

El conocimiento a crédito es una clase muy importante de conocimiento nocriterial. Es un conocimiento que no implica que los sujetos que lo poseen deban cualificarlo como tal conocimiento en virtud de la satisfacción de ciertos criterios. Y tampoco implica que deba hacerse tal cualificación antes de poseerlo. Es, además, un conocimiento no-criterial muy distinto del conocimiento no-criterial que podemos encontrar en el pragmatismo, en el utilitarismo o en la concepción del conocimiento como algo no-analizable. $Y$ es un conocimiento no-criterial capaz de asumir cualquier tipo de ascenso epistémico reflexivo. ${ }^{37}$

Si el conocimiento característicamente humano aspira a la reflexión, y si en la reflexión acabamos siempre encontrando un conocimiento a crédito, entonces podemos decir que todo nuestro conocimiento acaba siendo un conocimiento a crédito. ${ }^{38}$

Esto no impide que muchas de nuestras creencias se cualifiquen también como conocimientos en un sentido criterial, que incluye la existencia de conexiones veraces con el entorno. Esto es un hecho. Sin embargo, nuestro conocimiento acaba siempre siendo un conocimiento a crédito. En último término, nuestra imagen más global sobre la realidad y sobre nosotros mismos es un conocimiento a crédito. Es un conocimiento basado en el ejercicio virtuoso de nuestras capacidades para delegar y aplazar la justificación.

\footnotetext{
${ }^{37}$ Nuestra propuesta debe distinguirse de lo que a veces se ha llamado "The Credit View of Knowledge". Según este enfoque, las atribuciones de conocimiento pueden entenderse como atribuciones de crédito epistémico en el sentido de que cabe definir "S conoce que p" como "S merece crédito para creer con verdad que p". No estoy manteniendo esto. Incluso aunque las atribuciones de conocimiento impliquen atribuciones de crédito epistémico en este sentido, no pienso que el conocimiento pueda definirse como un tipo de atribución (de cierto crédito epistémico). Lo que estoy sosteniendo es que todo nuestro conocimiento es, en último término, un conocimiento a crédito en el sentido de que acaba apoyándose en ciertas capacidades virtuosas para delegar y aplazar la justificación.

${ }^{38}$ Hay conexiones interesantes entre el enfoque presentado en este trabajo y el enfoque defendido por Foley (1993). Ambos sugieren concepciones no-criteriales del conocimiento. Y ambos sugieren que nuestro conocimiento se desarrolla, y debe desarrollarse, sin ninguna red de seguridad en el trasfondo (en la propia terminología de Foley, “without a net"). Así mismo, ambos enfoques pondrían énfasis en la preservación de la espontaneidad epistémica. Sin embargo, nuestra propuesta es mucho menos "egocéntrica" que la de Foley. Las capacidades para "delegar" y para "aplazar" no son precisamente rasgos egocéntricos.
} 
¿Podemos decir algo más sobre estas capacidades? ¿Podemos decir algo más sobre cómo pueden constituirse en virtudes epistémicas? Un inicio de análisis debería contar al menos con los siguientes elementos:

(i) Las capacidades reflexivas para delegar y aplazar la justificación no serían virtudes fácilmente analizables como ligadas a facultades específicas, como seguramente lo sean las capacidades vinculadas a la percepción sensorial, la memoria o el razonamiento (esas capacidades reflexivas no serían "faculty-virtues"). Pero tampoco serían virtudes ligadas al carácter de un individuo particular como lo serían, por ejemplo, la constancia, la claridad o la amplitud de miras (tampoco serían "trait-virtues"). Serían virtudes epistémicas reflexivas que nos vinculan esencialmente a una comunidad y al desarrollo futuro de nuestra actividad epistémica.

(ii) Sin embargo, como hemos dicho, esas capacidades definirían el conocimiento característicamente bumano, un conocimiento que quiere ser reflexivo y que en último término no puede serlo en un sentido criterial.

(iii) Esas capacidades tendrían un poder estructurante crucial en relación a la unidad del resto de virtudes epistémicas. Constituirían el fundamento de todas las demás virtudes epistémicas que identificamos reflexivamente.

(iv) Y harían esto a través de procesos complejos en los que se fijaría de manera combinada y dinámica, respecto a los estados relevantes de conocimiento, quién podría esperarse que llevara a cabo las justificaciones pertinentes y cuándo podría esperarse que se lleven a cabo.

La reflexión tiene un enorme poder estructurante respecto a la totalidad del conocimiento humano. Pero el conocimiento reflexivo nos inclina hacia la no-criterialidad. Y una parte crucial de ese poder estructurante se deriva de un ejercicio virtuoso de nuestras capacidades para delegar y aplazar la justificación.

Como ya indicábamos al principio de nuestro trabajo, no podemos repetir aquí el movimiento de recuperación criterial que podemos hacer reflexivamente con el conocimiento animal. Aunque reflexivamente encontremos criterios (métodos, procedimientos, fuentes veraces, etc.) en relación al conocimiento animal, todo esto acaba transformado cuando el conocimiento reflexivo se amplía e intenta integrar circularidades virtuosas. En última instancia, acabamos fuera del juego de la criterialidad.

Que bajo un amplio examen reflexivo todo el conocimiento humano acabe siendo un conocimiento a crédito, y que la "autosustentación" ofrecida por las circularidades epistémicas acabe requiriendo inevitablemente capacidades virtuosas 
para delegar y aplazar la justificación, entraña que estas capacidades deban ser entendidas como procesos estructurantes que intervienen en la constitución de cualquier virtud epistémica. El que reflexivamente consideremos que una capacidad cognitiva constituye una virtud epistémica entraña poner en juego conocimientos basados en la delegación y el aplazamiento de ciertas justificaciones. El objetivo de tal delegación y aplazamiento es fijar, de algún modo, sin duda muy combinado y dinámico, un "quién" y un "cuándo" en las justificaciones que sirven de base a nuestro conocimiento reflexivo.

Pero la delegación y el aplazamiento de la justificación no sólo interviene en la configuración de la unidad de nuestras virtudes epistémicas. Esas capacidades también intervienen en la configuración de la unidad de nuestras virtudes epistémicas y noepistémicas. La razón es obvia. El carácter virtuoso completo de nuestras virtudes epistémicas depende de la manera cómo contribuyan a nuestra felicidad global.

Lo que podríamos llamar "felicidad epistémica" ha de ser un componente de nuestra felicidad global. Pero no en el sentido de que las fuentes de la felicidad epistémica simplemente se sume a otras fuentes de felicidad, sino en el sentido de podemos llamar "felicidad epistémica" a la contribución epistémica a nuestra felicidad global. ${ }^{39}$

Creo que todo esto puede vislumbrarse al final de algunos senderos que parten del camino conceptual emprendido por la epistemología de Ernest Sosa.

En los últimos trabajos de Sosa, se producen algunos cambios respecto a sus anteriores planteamientos. Podemos prestar atención, por ejemplo, a Sosa (2010) y Sosa (2015). Se propone entender la normatividad epistémica como un tipo de "normatividad de la actuación" (normatividad de las performances). Y se analizan con gran detalle los aspectos agentivos y normativos de los fenómenos epistémicos. ¿Hay cambios sustantivos que afecten sensiblemente a las consideraciones que hemos estado haciendo en nuestro trabajo? Creo que no. Creo que estos trabajos nos hacen incluso alejarnos aun más de una concepción criterial del conocimiento.

Veamos un poco más de cerca esos cambios. El modelo normativo que ofrece Sosa (2010) es elegante y convincente. Tener creencias es una actividad. Y su normatividad es la propia de las actuaciones (performances). Nuestras creencias deben

\footnotetext{
${ }^{39}$ El que las virtudes de un sujeto han de integrarse formando una peculiar "unidad orgánica" puede imponer fuertes condiciones sobre lo que por separado podrían constituir buenos modelos explicativos y evaluativos tanto respecto al conocimiento animal como respecto al conocimiento reflexivo. Sobre esta cuestión aún pueden aguardar sorpresas en el campo de la epistemología basada en la virtud.
} 
acertar en su objetivo, que es dar en el blanco de la verdad. También deben ser tenidas ejerciendo con destreza una cierta competencia epistémica, una cierta virtud. Por último, deben ser aptas en el sentido de que su acierto, su ser verdaderas, ha de manifestar esa competencia o virtud. Los rasgos que convierten una creencia en conocimiento son estos tres: acierto, destreza y aptitud. ${ }^{40}$

Esto es así para el conocimiento animal, para el conocimiento generado por las creencias de primer orden. Pero el conocimiento reflexivo, aún incorporando el conocimiento animal, va mucho más allá. El conocimiento reflexivo requiere meta-aptitud: creer aptamente que la creencia de primer orden es apta. Expliquemos mejor esta importante idea. Hay conocimiento reflexivo cuando se cree con aptitud (lo cual incluye acierto y destreza para esas creencias de orden superior) que un cierto conocimiento animal, o en cualquier caso conocimiento de orden más bajo, es realmente apto (lo cual incluye acierto y destreza para esas creencias de orden inferior).

El conocimiento característicamente humano quiere estar siempre acompañado de reflexión. Tal conocimiento reflexivo entraña un mayor control racional sobre nuestras creencias. Esto eleva su estatus epistemológico. Y hace que nuestro conocimiento sea un "pleno conocimiento". ${ }^{41}$

En Sosa (2015) encontramos también abundantes análisis del papel de la agencia y del contexto social en la creencia y en el juicio, sobre todo en conexión con la evaluación epistémica. Y se ofrecen propuestas generales acerca de lo que puede ser la evaluación normativa de una actuación, y de cómo enfocar la comprensión filosófica en este campo. ${ }^{42}$

\footnotetext{
${ }^{40}$ En este libro, Sosa (2010) introduce un modelo normativo para el conocimiento constituido por estos tres rasgos: Accuracy (acierto), Adroitness (destreza) y Aptness (aptitud). Abreviadamente, suele ya denominarse modelo AAA.

${ }^{41}$ Knowing Full Well es justamente el título de Sosa (2010), traducido al castellano por Modesto M. Gómez-Alonso con el título Con pleno conocimiento. Véase la interesante introducción que se hace en esta traducción de la epistemología de Sosa.

${ }^{42} \mathrm{La}$ obra de Sosa ha ido generando un interés creciente durante los últimos años. Y se multiplican los seminarios, congresos y publicaciones sobre sus propuestas. Muy centrados en los trabajos de Sosa, cabe destacar: Greco (ed.) (2004), Turry (ed.) (2013), Bahr y Seidel (eds.) (2016), Fernández (ed.) (2017) y VVAA (2017). Por otro lado, Sosa ha contribuido decisivamente a que la epistemología basada en la virtud se haya ido poco a poco constituyendo en un campo de desarrollo en continua y creciente actividad dentro del panorama de la filosofía contemporánea. Una muestra de ello la ofrecen: Alfano (ed.) (2015), Axtell (1997), Axtell (ed.) (2000), DePaul y Zagzebski (eds.) (2003), Fairweather y Zagzebski (eds.) (2001), Greco (1992), Greco (1993), Greco (2002), Greco (2010), Greco y Turri (eds.) (2012), Hookway (1994),
} 
¿Añaden algo diferente estos nuevos enfoques a la necesidad de un conocimiento reflexivo capaz de dar un sentido virtuoso a algunas circularidades? Si acaso, sólo se amplía aún más el contexto. Sigue manteniéndose intacta la aspiración a un amplio conocimiento reflexivo y la inevitabilidad de integrar en él circularidades epistémicas que puedan llegar a considerarse fuentes virtuosas de conocimiento. Pero ahora, la normatividad epistémica está indisolublemente mezclada con la normatividad práctica.

Tal vez algunos de los ejemplos empleados por Sosa (2010) nos lleven a pensar en un modelo simplemente deportivo de las virtudes epistémicas. Se compara allí el sujeto epistémico con un arquero diestro que sabe dar con sus creencias en el blanco de la verdad. Y de sus aciertos como aptos en la medida en que son resultado de su destreza. Pero también está la meta-aptitud de Diana la Cazadora, la diosa, consistente en saber elegir adecuadamente los objetivos. Y una meta-meta-aptitud, propia ya de nosotros, seres humanos, que consistiría en sopesar esas elecciones en relación a otros objetivos no exclusivamente epistémicos. Cuando se considera todo esto, enseguida queda involucrada una comunidad epistémica y un horizonte temporal. Y el modelo deportivo de la virtud debe dejar paso a otros modelos. En particular, y más allá del modelo artesanal que ya involucra una comunidad y una perspectiva temporal, debe dejar paso a modelos éticos y a modelos políticos de la virtud.

El conocimiento, podemos decir, no es un módulo que se acopla a otros módulos. La reflexión nos obliga a tener en cuenta, volveré a decir de manera dinámica y combinada, todos los aspectos de nuestra vida. Y muy especialmente a los aspectos comunitarios y temporales. Sólo así podremos entender la "unidad de la virtud".

¿Qué hay del modelo religioso de la virtud? En cierta forma, ya lo tenemos. El conocimiento reflexivo promueve la sabiduría. Y el don más preciado de la sabiduría es la confianza que genera. La confianza que produce el hacernos cargo de nuestra situación. Aunque en ella encontremos azar y suerte.

Entender el conocimiento como una cuestión de confianza nos libra de los avatares del azar o de la suerte. El azar o suerte desconocida no puede mermar nuestra confianza. Y el azar o suerte que vamos descubriendo, si no nos es totalmente adversa, y justamente eso esperamos, puede ser completamente asumible con una perspectiva adecuada.

Kvanvig (1992), Montmarquet (1993), Steup (ed.) (2001) y Zagzebski (1996). 


\section{COnClusiones}

Debemos confiar en nuestras fuerzas epistémicas porque necesitamos confiar en algo y porque sólo podemos confiar en esas fuerzas. Nos adherimos a aquello en lo que únicamente podemos confiar. Y nuestra adhesión es normativa.

¿Por qué necesitamos hacerlo? ¿Por qué necesitamos confiar en algo? Una buena respuesta es la siguiente: no aguantamos el escepticismo radical. No se trata de que sea auto-contradictorio afirmar que el conocimiento en general sea imposible. ${ }^{43}$ Aunque esto sea verdad, no es una verdad demasiado interesante. De lo que realmente se trata es de que no podemos vivir sin confiar en un gran número de nuestras creencias. Simplemente, necesitamos confiar en algo. A veces, muy explícitamente.

Es inevitable un mínimo de confianza epistémica. Y la encontramos en el propio pirronismo. Esta variedad escéptica criticaba al escepticismo académico por mantener incoherentemente que el conocimiento es imposible. Para los pirrónicos, este escepticismo sería tan dogmático como las epistemologías del estoicismo o del neoplatonismo. La alternativa pirrónica consiste en enseñar a prescindir de la epistemología. Los problemas epistemológicos son simplemente pseudo-problemas. No hay de qué preocuparse. El ideal pirrónico es una vida sin las angustias que producen los problemas epistemológicos. Con todo, el pirronismo se empeña en promover la investigación. Este es un tema constante en sus escritos. Y constituye uno de los aspectos más paradójicos del pirronismo. ¿Cómo es posible renegar de la epistemología y, a la vez, promover la investigación? ¿Por qué a pesar de mostrar la desconfianza más extrema sobre cualquiera de la creencias que podemos llegar a tener acerca de lo que sea el conocimiento cabe afirmar que debemos seguir investigando?

Una forma plausible de conciliar ambos extremos es interpretando la afirmación de que debemos seguir investigando como una expresión de la necesidad de confianza epistémica. Debemos seguir investigando porque queremos tener confianza epistémica en algo.

$\mathrm{El}$ argumento que inicia estas conclusiones se apoya en esta idea. Porque queremos confiar epistémicamente en algo, y porque sólo podemos tener esa confianza en nuestras propias fuerzas epistémicas, debemos confiar en ellas. Y si estas fuerzas acaban apoyándose en una comunidad y en el futuro, debemos confiar en la comunidady en el futuro.

\footnotetext{
${ }^{43}$ Esto incluiría no poder conocer el significado de lo que decimos, ni el significado de lo que pensamos.
} 
Estamos ante una significación filosófica del escepticismo mucho más profunda que la que consiste simplemente en hacernos pensar que tal vez haya siempre un vacio entre nuestro afán de conocimiento y el mundo en sí mismo. No se trata sólo de que haya un vacío. Ciertamente lo hay. Lo producen supuestos como los de un "demonio maligno cartesiano", ser "cerebros-en-cubetas", estar conectados a una Matrix envolvente, etc., o simplemente la ocasional intervención del azar o de la suerte. Pero no se trata sólo de esto. Se trata de que continuamente queremos dar saltos sobre ese vacío. Y se trata de que debemos confiar sólo en nuestras propias fuerzas para hacerlo.

Merece la pena "examinar nuestra vida". Una vida no reflexiva no es una vida auténticamente humana. Por ello, ampliamos los círculos que encontramos. Y buscamos nuevas perspectivas. $Y$ aunque nunca lleguemos a disfrutar de estados definitivos de confianza, aunque siempre dependamos de una comunidad epistémica y del tiempo, merece la pena estar comprometidos en estos procesos.

Acaso el conocimiento no sea realmente un estado sino un proceso. O mejor aún, una actividad. Si así fuera, entenderíamos por qué el conocimiento resulta indefinible como estado. Si así fuera, entenderíamos también por qué llega a involucrar por completo las comunidades epistémicas a las que pertenecemos y podemos pertenecer, así como a todos nuestros futuros "yoes".

El conocimiento como proceso incluiría además una sincera aceptación del falibilismo. Podemos estar equivocados en cualquiera de nuestras creencias. Siempre puede haber "demonios malignos cartesianos", siempre podemos ser "cerebros-en cubetas", siempre podemos estar en alguna Matrix. O simplemente, siempre puede defraudar nuestra confianza el azar o la suerte. Pero el falibilismo es sólo una parte de nuestra propuesta. Otra parte no menos importante, otra parte que equilibra la anterior, consiste en que debemos confiar plenamente en nuestras fuerzas epistémicas. Y no se trata simplemente de que podamos hacer esto. Es que debemos hacerlo.

\author{
Manuel Liz Gutiérrez. \\ Universidad de La Laguna \\ manuli_@gmail.com
}




\section{BIBLIOGRAFÍA}

Alfano, M. (ed.) (2015), Current Controversies in Virtue Theory, Nueva York, Routledge.

Axtell, G. (1997), "Recent work in Virtue Epistemology", American Pbilosophical Quarterly, 34, pp. 1-27.

- (ed.) (2000), Knowledge, Belief, and Character. Readings in Virtue Epistemology, Lanham, Rowman \& Littlefield.

BAHr, A., y M. SEIDEL (eds.) (2016), Ernest Sosa. Targeting His Pbilosophy, Dordrecht, Springer. Bonjour, J. (1985), The Structure of Empirical Knowledge, Cambridge, Harvard Univ. Press. BONJOUR, L., y E. SOSA (2003), Epistemic Justification. Internalism vs. Externalism, Foundations vs. Virtues, Oxford, Blackwell.

Broncano, F., y J. Vega (2009), "Las fuentes de la normatividad epistémica: Deberes, funciones, virtudes", en Quesada, D. (Coord.) (2009), cap. 3, pp. 77-110.

Chisholm, R. (1942), “The Problem of the Speckled Hen”, Mind, 51, 368-73.

- (1982), "The Problem of the Criterion", en The Foundations of Knowing, Minneapolis,

Univ. of Minnesota Press.

— (1989), Theory of Knowledge ( $3^{a}$ ed.), Englewood Cliffs, Prentice Hall.

Cohen, S. (2002), "Basic Knowledge and the Problem of Easy Knowledge", Philosophy and Phenomenological Research, vol LXV, $\mathrm{n}^{\circ}$ 2, 309-329.

Davidson, D. (1986), "A Coherence Theory of Truth and Knowledge", en E. LePore (ed.) Truth and Interpretation. Perspectives on the Pbilosophy of Donald Davidson, Londres, Blackwell, 1986, pp. 307-319.

DePaul, M. y L. ZagZEBSKi (eds.) (2003), Intellectual Virtue: Perspectives from Ethics and Epistemology, Oxford, Oxford Univ. Press.

DeRose, K. (2008), "Sosa, Safety, Sensitivity, and Skeptical Hypotheses", en J. Greco (ed.) Ernest Sosa and His Critics, Londres, Blackwell, 2008, cap. 3., 22-41.

DretSKe, F. (1981), Knowledge and the Flow of Information, Cambridge, MIT Press.

Engel, P. (2007), "La verdad y el objetivo de la creencia”, en D. Pérez Chico y M. Barroso (eds.) Pluralidad de la filosofía analitica, Madrid, Plaza y Valdés, 2007.

FAirweATHER, A., y L. ZAGZEBSKi (eds.) (2001), Virtue Epistemology: Essays on Epistemic Virtue and Responsibility, Oxford, Oxford Univ. Press.

Fernández, M. A. (ed.) (2017), Performance Normativity, Oxford, Oxford Univ. Press (próxima publicación).

FOLEY, R. (1992), Working without a net, Princeton, Princeton Univ. Press.

Goldman, A. (1986), Epistemology and Cognition, Cambridge, Harvard Univ. Press.

GreCO, J. (1992), "Virtue Epistemology”, en Dancy y Sosa (eds.) (1992).

- (1993) "Virtues and Vices of Virtue Epistemology", Canadian Journal of Philosophy, 23, pp. 413-432.

- (2002), "Virtues in Epistemology", en Moser (ed.) (2002).

— (ed.) (2004), Ernst Sosa and His Critics, Oxford, Blackwell. 
Greco, J. (2010), Achieving Knowledge. A virtue-theoretic Account of Epistemic Normativity, Cambridge, Cambridge Univ. Press.

Greco, J. y J, Turri (eds.) (2012), Virtue Epistemology, Cambridge, MIT Press.

Groff, R., y J. Greco (eds.) (2013), Powers and Capacities in Philosophy. The New Aristotelism, London, Routledge.

Hales, S. (2015a), "A Problem for Moral Luck", Philosophical Studies, 172:9, 2385-2403.

- (2015b), "Synchronic and Diachronic Luck", en M. Vázquez y M. Liz (eds.) Temporal

Points of View: Subjective and Objective Aspects, Berlin, Springer, 2015, 255-263.

- (2016), "Why Every Theory of Luck is Wrong", Nous, 50:3, 490-508.

HOOKWAY, C. (1994), "Cognitive Virtues and Epistemic Evaluation”, International Journal of Philosophical Studies, 2, pp. 211-27.

Kennedy, R. (1993), "Professor Chisholm and the Problem of the Speckled Hen", Journal of Philosophical Research, 18, pp. 143-147.

Kvanvig, J. (1992), The Intellectual Virtues and the Life of the Mind: On the Place of the Virtues in Contemporary Epistemology, Savage, Rowman and Littlefield.

LeHrer, K. (1986), "The Coherence Theory of Knowledge”, Philosophical Topics, 14(1), pp. $5-25$.

LIZ, M. (2003), Justificary explicar. La justificación epistémica como un tipo de explicación naturalista guiada por la reflexión, La Laguna, Servicio de Publicaciones de la Univ. de La Laguna.

MARKiE, P. (2009), "Classical Foundationalism and Spekled Hens", Philosophy and Phenomenological Research, 79(1), pp. 190-206.

McIntyre, A. (1981), After Virtue. A Study in Moral Theory, Indiana, Univ. of Notre Dame Press.

Montmarquet, J. (1993), Epistemic Virtue and Doxastic Responsibility, Lanham, Rowman $\&$ Littlefield.

NozICK, R. (1981), Philosophical Explanations, Cambridge, Harvard Univ. Press.

Nussbaum, M. (1986), The Fragility of Goodness: Luck and Ethics in Greek Tragedy and Philosophy, Nueva York, Cambridge Univ. Press.

Poston, T. (2007), "Acquaintance and the problem of the Speckled Hen", Philosophical Studies, 132(2), pp. 331-46.

SosA, E. (1991), Knowledge in Perspective, Selected Essays in Epistemology, Cambridge, Cambridge Univ. Press.

- (2007), A Virtue Epistemology. Apt Belief and Reflective Knowledge, vol. I, Oxford, Oxford Univ. Press.

- (2008), "How to Defeat Opposition to Moore", en E. Sosa, J. Kim, J. Fantl y M. McGrath (eds.) Epistemology: An Ontology, Malden, Blackwell, 2008.

- (2009), Reflective Knowledge. Apt Belief and Reflective Knowledge, vol. II, Oxford, Oxford Univ. Press.

- (2010), Knowing Full Well, Princeton, Princeton Univ. Press [Trad. Castellana: Con Pleno Conocimiento, Zaragoza, Prensas de la Univ. de Zaragoza, 2014. Introducción, traducción y notas de Modesto M. Gómez-Alonso]. 
SosA, E. (2015), Judgment and Agency, Oxford, Oxford Univ. Press

- y B. STROUD (1994), "Philosophical Scepticism", Proceedings of the Aristotelian Society, Supplementary Volumes, Vol. 68, pp. 263-307.

STEup, M. (ed.) (2001), Knowledge, Truth, and Duty. Essays on Epistemic Justification and the Ethics of Belief, Oxford, Oxford Univ. Press.

Stroud, B. (1984), The Significance of Philosophical Scepticism, Oxford, Oxford Univ. Press.

- (2002), Understanding Human Knowledge, Oxford, Oxford Univ. Press.

TURry, J. (ed.) (2013), Virtuous Thoughts: The Philosophy of Ernest Sosa, Dordrecht, Springer.

VVAA (2017), The Philosophy of Ernest Sosa, número especial de Synthese (próxima publicación).

Williamson, T. (2000), Knowledge and Its Limits, Oxford, Oxford University Press.

Zagzebski, L. (1996), Virtues of the Mind. An Inquiry into the Nature of Virtue and Ethical Foundations of Knowledge, Cambridge, Cambridge Univ. Press.

Zalabardo, J. (2005), "Skepticism and the Problem of Easy Knowledge", The Philosophical Review, 114, 33-61.

- (2014), "Cómo sé que no soy un cerebro en una cubeta”, en J. Zalabardo Conocimiento y escepticismo. Ensayos de epistemología, México, UNAM, Instituto de Investigaciones Filosóficas, 2014, cap. IV. 